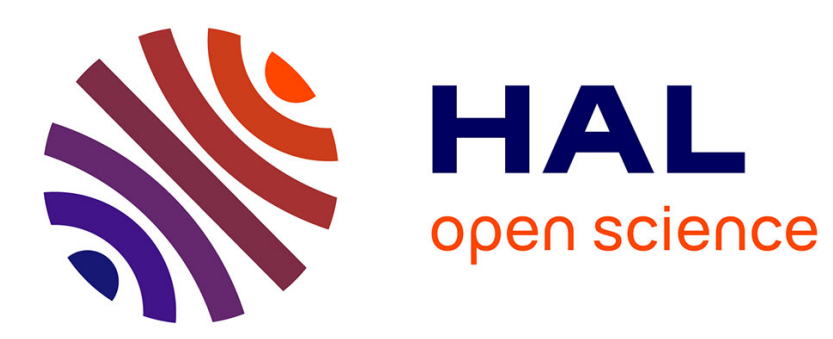

\title{
Access to Isothiazolones from Simple Acrylamides by Pd-Catalyzed C-H Bond Activation
}

Mu-Yi Chen, Xavier Pannecoucke, Philippe Jubault, Tatiana Besset

\section{To cite this version:}

Mu-Yi Chen, Xavier Pannecoucke, Philippe Jubault, Tatiana Besset. Access to Isothiazolones from Simple Acrylamides by Pd-Catalyzed C-H Bond Activation. Journal of Organic Chemistry, In press, 84 (20), pp.13194-13202. 10.1021/acs.joc.9b01872 . hal-02267936

\section{HAL Id: hal-02267936}

\section{https://hal-normandie-univ.archives-ouvertes.fr/hal-02267936}

Submitted on 5 Sep 2019

HAL is a multi-disciplinary open access archive for the deposit and dissemination of scientific research documents, whether they are published or not. The documents may come from teaching and research institutions in France or abroad, or from public or private research centers.
L'archive ouverte pluridisciplinaire $\mathbf{H A L}$, est destinée au dépôt et à la diffusion de documents scientifiques de niveau recherche, publiés ou non, émanant des établissements d'enseignement et de recherche français ou étrangers, des laboratoires publics ou privés. 


\title{
Access to Isothiazolones from Simple Acrylamides by Pd-Catalyzed C-H Bond Activation
}

\author{
Mu-Yi Chen, Xavier Pannecoucke, Philippe Jubault, Tatiana Besset* \\ Normandie Univ, INSA Rouen, UNIROUEN, CNRS, COBRA (UMR 6014), 76000 Rouen, \\ France. \\ E-mail: tatiana.besset@insa-rouen.fr
}

\begin{abstract}
A new methodology was developed to access isothiazolone derivatives from simple acrylamides by transition metal catalyzed $\mathrm{C}-\mathrm{H}$ bond functionalization. This Pd-catalyzed reaction using an electrophilic SCN source offered an efficient tool to access a panel of functionalized isothiazolone derivatives (21 examples, up to $71 \%$ yield).
\end{abstract}

Over the last years, the development of new catalytic systems to tackle unaddressed synthetic issues aroused the curiosity of the scientific community to imagine and design new tools. As part of them, the direct functionalization of a simple $\mathrm{C}-\mathrm{H}$ bond appeared as an efficient way to accomplish this task and a panel of transition metal catalyzed transformations is now available. ${ }^{1}$ With these atom- and step-economical synthetic pathways, unprecedented retrodisconnections were achieved, offering original synthetic routes towards the construction of more complex molecules. ${ }^{2}$ However, it is worth mentioning that compared to the tremendous advances made for the functionalization of aromatic compounds, the transition metal catalyzed functionalization of vinylic derivatives by $\mathrm{C}-\mathrm{H}$ bond activation is rather limited. ${ }^{3}$ In this context, we turned our attention to the synthesis of isothiazolone derivatives. These underexplored $N, S$ heterocycles are of high interest and found applications as antimicrobials, in paint formulation as well as in hair care products and shampoos. For instance, $\mathrm{N}$-phenylisothiazolones with various substituents at $\mathrm{C} 4$ and $\mathrm{C} 5$ positions turned out to be good candidates as inhibitors of the histone acetyltransferase enzymes. ${ }^{4,5}$ Usually, $N$-substituted isothiazolones are prepared by different approaches (Scheme 1). Pioneer works from the group of Lewis in 1971, followed by several other research groups, relied on the cyclization of $N, N^{\prime}$-bis-aryl-3,3'dithiodipropionamide derivatives in the presence of chlorine gas, $\mathrm{SOCl}_{2}$ or $\mathrm{SO}_{2} \mathrm{Cl}_{2}$ (Scheme 1 , eq. 1). ${ }^{6}$ Isothiazolones were also prepared from 3-aroylpropionic amides upon reaction with thionyl chloride (Scheme 1, eq. 2). ${ }^{7}$ An alternative pathway relying on an oxidative addition with iodine under basic conditions was depicted by Petraitis and co-workers starting from 3- 
aryl-3-mercapto-propenamide derivatives (Scheme 1, eq. 3). ${ }^{8}$ Finally, complementary approaches were developed to access $N$-substituted-5-aryl/alkyl-substituted-isothiazolones. In 2009, the group of McDonald developed a Pummerer-like type reaction for the synthesis of $\mathrm{N}$ aryl-5-aryl-isothiazolone and $\mathrm{N}$-aryl-5-alkyl-isothiazolone compounds (Scheme 1, eq. 4). ${ }^{4}$ In 2017, Reddy and co-workers reported a thiocyanation/intramolecular decyanative cyclization of ynamides leading to the corresponding $N$-substituted-5-phenyl-isothiazolones (5 examples, Scheme 1, eq. 5). ${ }^{9}$ Compared to traditional routes, the development of alternative synthetic pathways to provide an access to various isothiazolone derivatives with good diversity at the C4 position from simple starting materials is appealing.

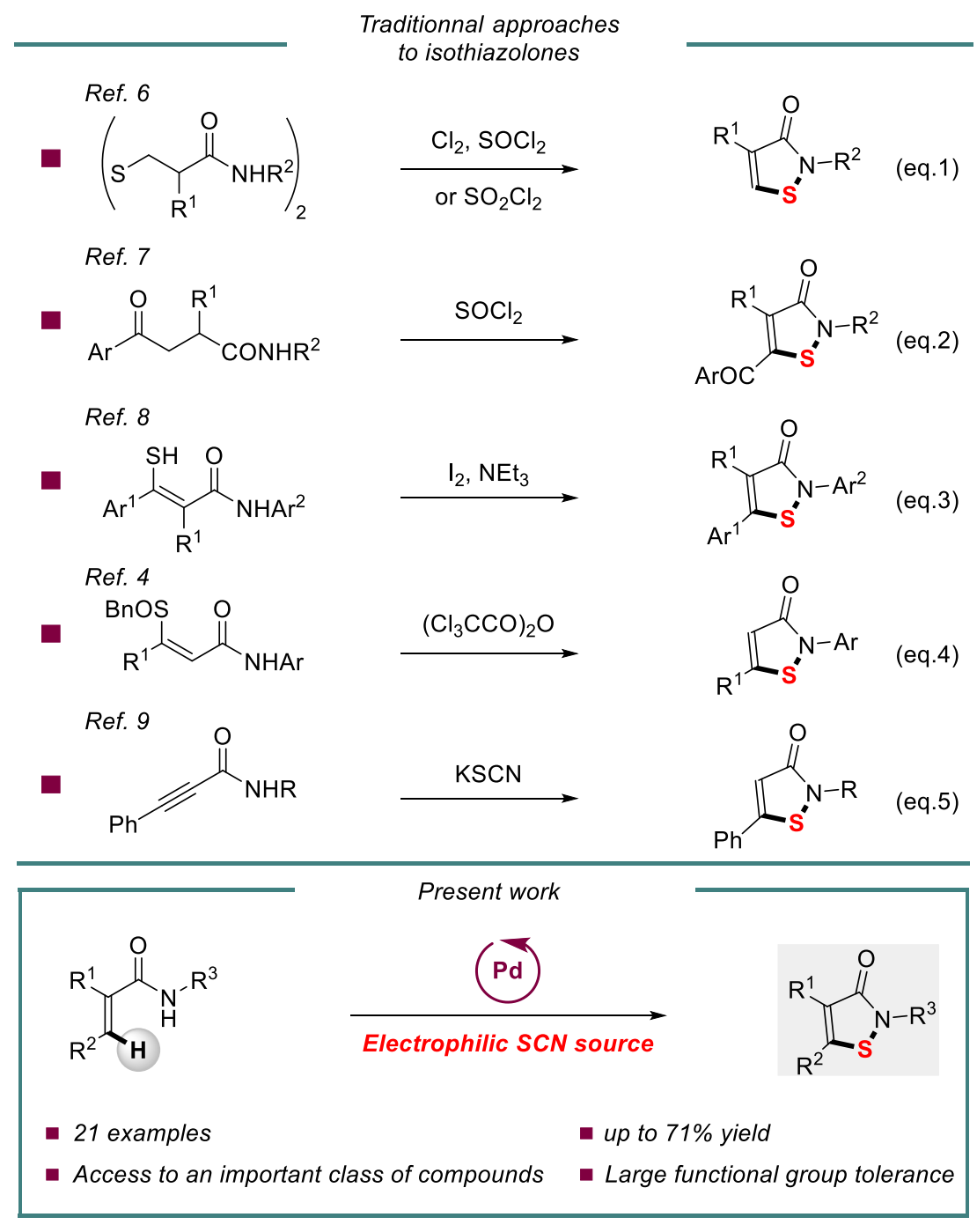

Scheme 1. Synthesis of isothiazolone derivatives: traditional approaches and present work.

Inspired by our recent investigations on the functionalization of vinylic $\mathrm{C}-\mathrm{H}$ bond by transition metal catalysis, ${ }^{10}$ we sought that the reaction of an acrylamide with an electrophilic SCN source in the presence of a Pd-catalyst could afford the isothiazolone backbone selectively. Herein, we 
report the first Pd-catalyzed synthesis of 4-substituted and 4,5-disubstituted isothiazolones from acrylamide derivatives.

We began our investigation using the acrylamide 1a in presence of the electrophilic SCN source I under Pd catalysis at $80{ }^{\circ} \mathrm{C}$ under air. The corresponding isothiazolone derivative 2a was selectively obtained in a $44 \%$ NMR yield. ${ }^{11}$ Note that no product resulting from the thiocyanation reaction was detected. ${ }^{12}$ First, several Pd-catalysts were tried (Table 1, entries 14) and $\mathrm{PdCl}_{2}$ turned out to be the most efficient one in this transformation. Importantly, in the absence of Pd-catalyst, no reaction occurred showcasing with this control experiment its key role in the synthesis of isothiazolones (Table 1, entry 5). When the reaction was performed under inert atmosphere or in the presence of a catalytic amount of PivOH (25 mol\%), similar results were obtained (Table 1, entries 6 and 7). When the temperature was lowered to $60{ }^{\circ} \mathrm{C}$, 2a was isolated in only $51 \%$ yield (Table 1, entry 8 ). On the contrary, an increase of the temperature to $100{ }^{\circ} \mathrm{C}$ and $120{ }^{\circ} \mathrm{C}$ gave similar results, the reaction at $100{ }^{\circ} \mathrm{C}$ being the most efficient one (Table 1, entries 9 and 10). Finally, when other solvents were evaluated such as 1.4-dioxane, toluene and DCE, a shutdown of the reactivity or lower yields were obtained (Table 1, entries 11-13). 
Table 1. Optimization of the reaction conditions ${ }^{a}$<smiles>CC(C)(C)c1ccc(C(=CCO)C(=O)Nc2cccc3cccnc23)cc1</smiles>

$1 \mathbf{a}$<smiles>N#SN1C(=O)c2ccccc2C1=O</smiles>

I

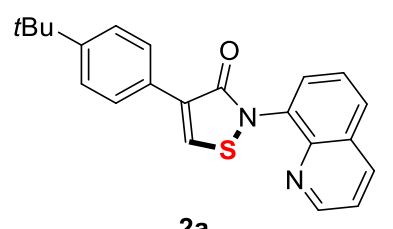

2a

\begin{tabular}{|l|l|l|l|l|}
\hline entry & catalyst & $\begin{array}{l}\mathrm{T} \\
\left({ }^{\circ} \mathrm{C}\right)\end{array}$ & solvent & $\begin{array}{l}{ }^{1} \mathrm{H} \text { NMR } \\
\text { yield }(\%)\end{array}$ \\
\hline 1 & $\mathrm{Pd}(\mathrm{OAc})_{2}$ & 80 & DMF & 44 \\
\hline 2 & $\mathrm{PdBr}_{2}$ & 80 & DMF & 45 \\
\hline 3 & ${\mathrm{Pd}\left(\mathrm{CH}_{3} \mathrm{CN}\right)_{2} \mathrm{Cl}_{2}}^{2}$ & 80 & DMF & 55 \\
\hline 4 & $\mathrm{PdCl}_{2}$ & 80 & DMF & $81,63^{b}$ \\
\hline 5 & $\mathrm{None}^{2}$ & 80 & DMF & $\mathrm{NR}$ \\
\hline $6^{c}$ & $\mathrm{PdCl}_{2}$ & 80 & DMF & $78,61^{b}$ \\
\hline 8 & $\mathrm{PdCl}_{2}$ & 80 & DMF & $78,60^{b}$ \\
\hline $\mathbf{P d C l}$ & & 60 & DMF & $62,51^{b}$ \\
\hline 10 & $\mathbf{P d C l}_{2}$ & $\mathbf{1 0 0}$ & DMF & $\mathbf{8 7 , \mathbf { 7 0 } ^ { b }}$ \\
\hline 11 & $\mathrm{PdCl}_{2}$ & 120 & DMF & $87,69^{b}$ \\
\hline 12 & $\mathrm{PdCl}_{2}$ & 100 & $1.4-$ dioxane & $\mathrm{NR}$ \\
\hline 13 & $\mathrm{PdCl}_{2}$ & 100 & toluene & 55 \\
\hline
\end{tabular}

${ }^{a}$ Reaction conditions: $1 \mathrm{a}(0.1 \mathrm{mmol}), \mathrm{SCN}$ reagent $\mathbf{I}(0.3 \mathrm{mmol})$, catalyst $(10 \mathrm{~mol} \%)$, solvent $(0.1 \mathrm{M}), \mathrm{T}\left({ }^{\circ} \mathrm{C}\right), 16$ $\mathrm{h}$, air. Yields determined by ${ }^{1} \mathrm{H}$ NMR on the crude reaction mixture using 1.1.2.2-tetrachloroethane as an internal standard. ${ }^{b}$ Isolated yields. ${ }^{c}$ Under Ar atmosphere. ${ }^{d}$ Using $25 \mathrm{~mol} \%$ of PivOH as additive. PivOH $=$ pivalic acid. $\mathrm{DCE}=1.2$-dichloroethane. $\mathrm{NR}=$ no reaction.

With the best reaction conditions in hand, the scope of the transformation was investigated under Pd-catalysis. A panel of $\alpha$-aryl acrylamides were functionalized in moderate to good yields (Scheme 2). Acrylamides with arenes bearing electron-rich substituents (1a-e) and halogens (1g-i) at the para-position were converted into the corresponding $N$-quinolyl-4arylisothiazolones. The presence of the benzyl alcohol (compound 2d) was a key value added since not tolerated in the previously reported reaction conditions. ${ }^{9}$ The substitution pattern on the aromatic ring did not have any impact on the reaction outcome as observed with $\mathbf{2} \mathbf{b}, \mathbf{2} \mathbf{j}$ and $\mathbf{2 m}$ or $\mathbf{2 h}, \mathbf{2 k}$ and $\mathbf{2 n}$. The reaction was tolerant to various substituents such as halogens and 
$\mathrm{CF}_{3}$ group (2g-i, $\mathbf{2 k}$ and $\mathbf{2 n - 0}$ ), and no product resulting from a protodehalogenation reaction was detected. Note that the reaction was easily scaled up and $\mathbf{2 g}$ was synthesized in $65 \%$ yield on a $1 \mathrm{mmol}$ scale. Heteroaromatic ring was also tolerated as demonstrated with compound $\mathbf{2 r}$, although it was obtained in a somehow lower yield (45\%). The reaction was not restricted to $\alpha-$ aryl acrylamides and challenging $\alpha, \beta$-disubstituted acrylamides were also suitable substrates, offering an access to fully decorated isothiazolone derivatives. $\alpha$-Phenyl-trans-cinnamide $1 \mathbf{s}$ and $\alpha, \beta$-dimethyl acrylamide $\mathbf{1 t}$ were engaged in the standard reaction conditions. The transformation yielded the corresponding products $2 \mathrm{~s}$ and $\mathbf{2 t}$ in $49 \%$ and $51 \%$ yields, respectively, offering an access to other isothiazolone derivatives and demonstrating the synthetic utility of the reaction. Finally, when the amide derived from 8-amino-5methoxyquinoline was used as a directing group, the corresponding product $\mathbf{2} \mathbf{u}$ was obtained in $50 \%$ and $49 \%$ yields (on 0.2 and $0.5 \mathrm{mmol}$ scale, respectively). However, trans-cinnamide $\mathbf{1 v}$ was a reluctant substrate, which highlighted the complementary of our approach compared to some previous report. ${ }^{9}$ To gain more insight in the transformation, other directing groups were investigated. No reaction was observed with the amide derived from the $N$-methyl amide $\mathbf{1 w}$, highlighting the key role of the NH from the directing group in the reaction. Note that when the amide derived from the 2-(aminomethyl)pyridine $\mathbf{3}$ as well as tertiary amides $\mathbf{4}$ and $\mathbf{5}$ were used, no reaction occurred. Unfortunately, all attempts to cleave the directing group in the presence of CAN to get the free amide failed, and the starting material was fully recovered. 

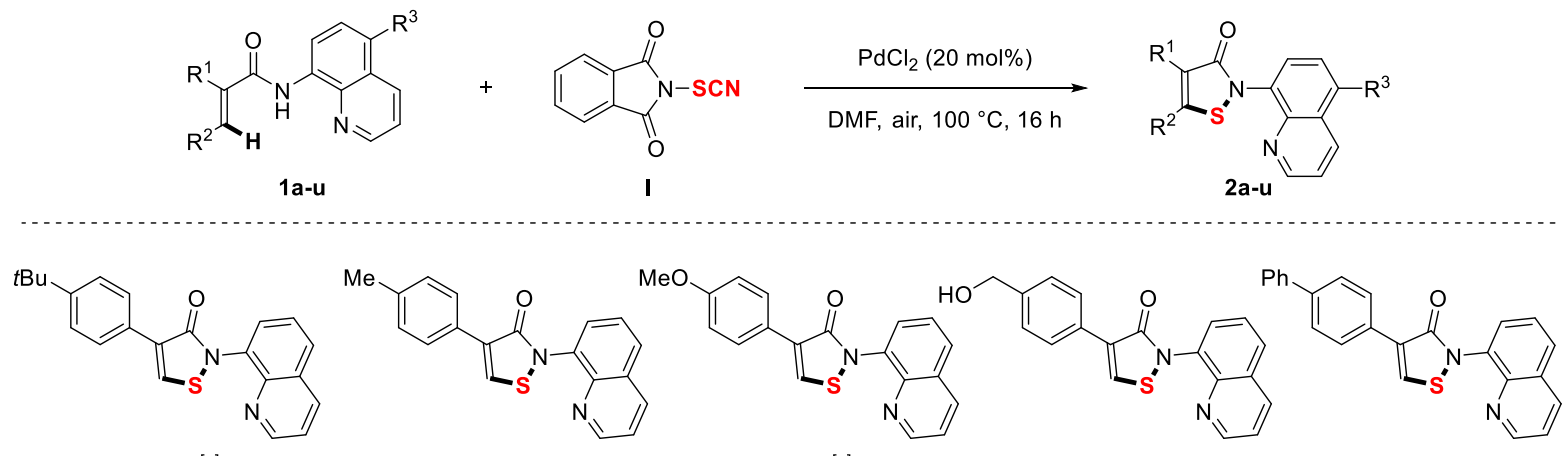

2a, $70 \%{ }^{[a]}$

2b, $59 \%$

2c, $52 \%$ [a]

2d, $41 \%$

2e, $50 \%$<smiles>O=c1c(-c2ccccc2)csn1-c1cccc2cccnc12</smiles>

2f, $62 \%$<smiles>O=c1c(-c2ccc(F)cc2)csn1-c1cccc2cccnc12</smiles>

2g, $71 \%, 65 \%[$ [b]<smiles>O=c1c(-c2ccc(Cl)cc2)csn1-c1cccc2cccnc12</smiles>

2h, $60 \%$<smiles>Cc1cccc(-c2csn(-c3cccc4cccnc34)c2=O)c1</smiles>

2i, $45 \%$

2j, $61 \%$<smiles>O=c1c(-c2cccc(Cl)c2)csn1-c1cccc2cccnc12</smiles><smiles>O=c1c(-c2cccc(C(F)(F)F)c2)csn1-c1cccc2cccnc12</smiles><smiles>Cc1ccccc1-c1csn(-c2cccc3cccnc23)c1=O</smiles><smiles>O=c1c(-c2ccccc2Cl)csn1-c1cccc2cccnc12</smiles>

2n, $62 \%$

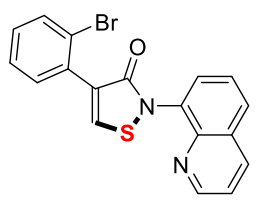

2o, $65 \%$

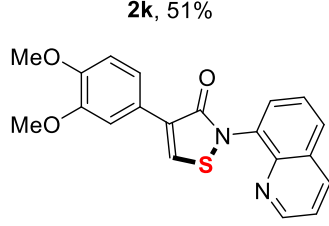

2I, $65 \%$<smiles>O=c1c(-c2ccc3c(c2)OCO3)csn1-c1cccc2cccnc12</smiles><smiles>O=c1c(-c2ccc(Cl)nc2)csn1-c1cccc2cccnc12</smiles>

2 r, $45 \%$

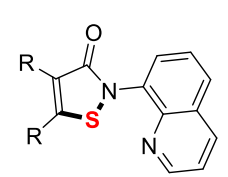

2s, $\mathrm{R}=\mathrm{Ph}, 49 \%$ 2t, $\mathrm{R}=\mathrm{Me}, 51 \%$

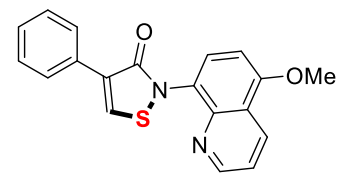

$2 u, 50 \%, 49 \%\left[{ }^{[c]}\right.$<smiles>CC/C=C(\C(=O)NCC)c1ccc(C)cc1</smiles><smiles>C=C(C(=O)NCCCN)c1ccccc1</smiles>

Scheme 2. Functionalization of acrylamides 1 into the corresponding isothiazolones 2. Reaction conditions: $1(0.2$ $\mathrm{mmol}), \mathbf{I}(0.6 \mathrm{mmol}), \mathrm{PdCl}_{2}(20 \mathrm{~mol} \%), \operatorname{DMF}(2 \mathrm{~mL}), 100{ }^{\circ} \mathrm{C}, 16 \mathrm{~h}$, air, isolated yields were given. [a] $10 \mathrm{~mol} \%$ of $\mathrm{PdCl}_{2}$ was used. [b] Reaction was performed on a $1 \mathrm{mmol}$ scale using 4 equivalents of $\mathbf{I}$. [c] Reaction was performed on a $0.5 \mathrm{mmol}$ scale.

In order to further demonstrate the synthetic utility of the products 2 , an additional transformation was conducted (Scheme 3). The selective chlorination of the 8-aminoquinoline part was realized, bringing functional group diversity on that part of the molecule too. Indeed, in the presence of 0.5 equivalent of TCCA, the functionalized isothiazolone 6 was isolated in $55 \%$ yield. 


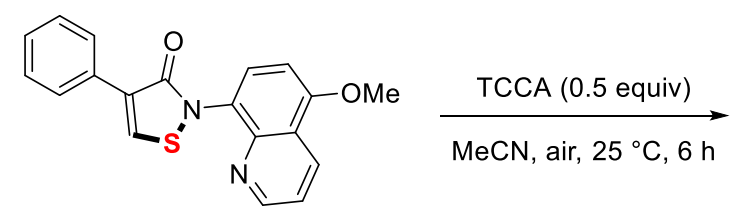

$2 u$

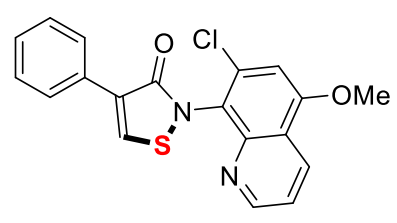

6, $55 \%$

Scheme 3. Selective chlorination reaction of the compound $\mathbf{2 u}$

Based on our previous work regarding the functionalization of olefins according to a C-H bond functionalization via Pd-catalysis ${ }^{10 \mathrm{c}}$ and the fact that the transformation did not occur without a Pd-catalyst (Table 1, entry 5), a possible mechanism was suggested (Scheme 4). Subsequent to the coordination of the $\mathrm{Pd}(\mathrm{II})$-catalyst with the bidentate directing group, which afforded the species A, the corresponding palladacycle (intermediate B) was formed. This process might occur via a concerted metalation-deprotonation step. In the presence of the electrophilic SCN source I, an oxidative addition reaction with the intermediate $\mathbf{B}$ led to a $\mathrm{Pd}(\mathrm{IV})$ species $\mathbf{C}$. Then, a final reductive elimination along with the protonation of the nitrogen atom of the amide regenerated the catalyst and afforded a putative intermediate $\mathbf{D}$, which quickly underwent an intramolecular decyanative cyclization to provide the desired isothiazolone $2{ }^{9}$

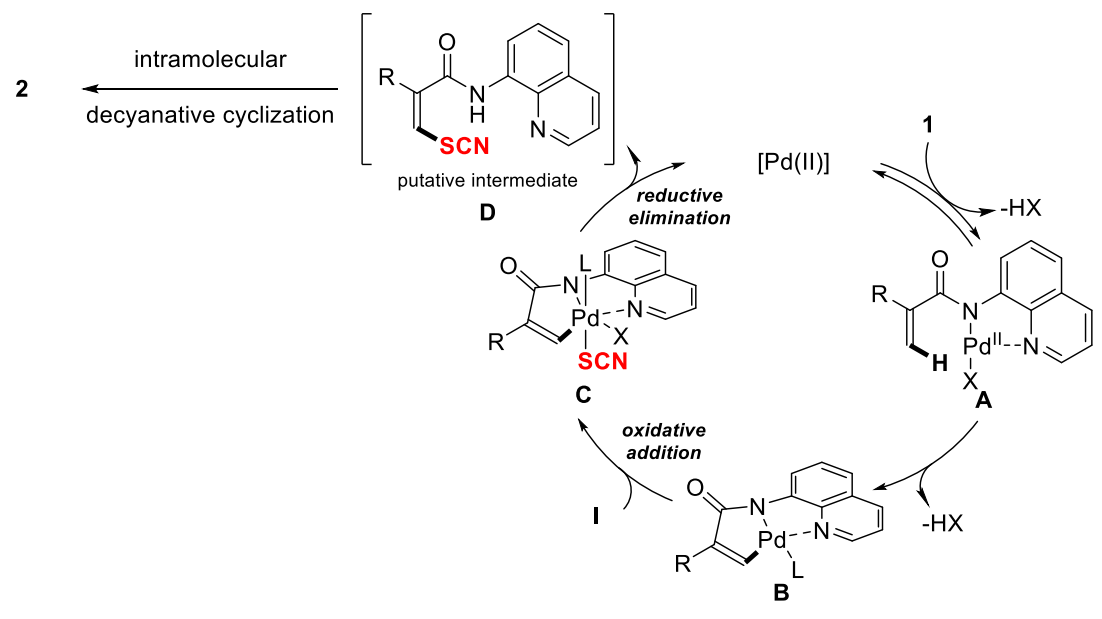

Scheme 4. Plausible catalytic cycle

\section{Conclusion}

In this study, an original synthesis of $N$-quinolyl-4-arylisothiazolones and $N$-quinolyl-4,5disubstituted isothiazolones was achieved under an air atmosphere. An array of heterocyclic compounds was obtained in moderate to good yields ( 21 examples, up to $71 \%$ yield) using simple and neutral reaction conditions. With this approach, not only 4-substituted but also 4,5- 
disubstituted isothiazolone derivatives were obtained. A post-functionalization reaction further demonstrated the synthetic utility of the depicted approach. We believe that this methodology will be of high interest for the scientific community, offering a straightforward access to molecules of interest, and demonstrating further how the direct $\mathrm{C}-\mathrm{H}$ bond functionalization might be used to build up more complex molecules.

\section{Experimental Section}

All reactions were carried out using oven dried glassware and magnetic stirring under an atmosphere of air unless otherwise stated. Reaction temperatures are reported as the temperature of the oil bath surrounding the vessel. Analytical thin layer chromatography was performed on silica gel aluminium plates with F-254 indicator and visualized by UV light (254 $\mathrm{nm}$ ) and/or chemical staining with a $\mathrm{KMnO}_{4}$ solution or a phosphomolybdic acid solution. Flash chromatography was performed on Merck silica gel (40-63 mesh) either by standard technique or by Biotage Isolera One Flash Purification System (gradient of solvents; PE = petroleum ether, $\mathrm{Et}_{2} \mathrm{O}=$ diethyl ether). ${ }^{1} \mathrm{H}$ NMR spectra were recorded on a Bruker DXP $300 \mathrm{MHz}$ spectrometer at $300.1 \mathrm{MHz},{ }^{13} \mathrm{C}$ spectra at $75.5 \mathrm{MHz},{ }^{19} \mathrm{~F} \mathrm{NMR}$ at $282.4 \mathrm{MHz}$. Chemical shifts $(\delta)$ are quoted in parts per million (ppm) relative to residual solvent peak for $\mathrm{CDCl}_{3}\left(\delta_{\mathrm{H}}=7.26\right.$ ppm; $\delta_{\mathrm{C}}=77.0 \mathrm{ppm}$; or relative to external $\left.\mathrm{CFCl}_{3}: \delta=0.0 \mathrm{ppm}\right)$. Coupling constants $(J)$ are quoted in Hz. The following abbreviations were used to show the multiplicities: s: singlet, d: doublet, dd: doublet of doublet, m: multiplet. High-Resolution Mass Spectra (HRMS) were recorded on Waters LCT Premier. Infrared spectra were recorded on a Perkin Elmer FT-IR spectrometer Paragon 100 (ATR); the wave numbers $(v)$ are quoted in $\mathrm{cm}^{-1}$. Melting points were recorded on Kofler bench and are uncorrected.

$\mathrm{PdCl}_{2}$ was purchased from Sigma-Aldrich Ltd. Dry DMF ( $N, N$-Dimethylformamide, in sealed bottle with molecular sieves) was purchased from Acros Organics Ltd. Reagent I was synthesized according to the literature procedure. ${ }^{13}$ Acrylamides $\mathbf{1 a},{ }^{10 \mathrm{c}} \mathbf{1 b},{ }^{14} \mathbf{1 c},{ }^{10 \mathrm{c}} \mathbf{1 e - 1 f},{ }^{10 \mathrm{c}}$

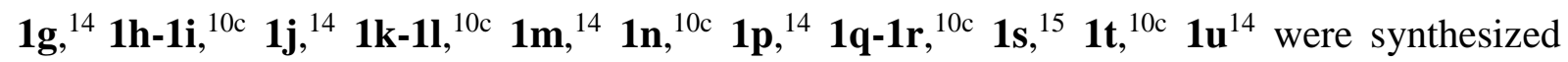
according to the preview reports in the literature. Starting materials $\mathbf{1 v},{ }^{16} \mathbf{3},{ }^{16} \mathbf{4}^{17}$ and $\mathbf{5}^{10 \mathrm{~b}}$ were prepared according to literature procedures.

\section{General Procedures for the Preparation of the Starting Materials 1.}

General procedure for the synthesis of 2-aryl-N-(quinolin-8-yl)acrylamides 1a-1e, 1g-1r. Oxalyl chloride $\left(0.94 \mathrm{~mL}, 11 \mathrm{mmol}, 1.1\right.$ equiv) was slowly added at $0{ }^{\circ} \mathrm{C}$ to a solution of the corresponding acid (10 mmol, 1.0 equiv) and DMF (3 drops) in freshly distilled $\mathrm{CH}_{2} \mathrm{Cl}_{2}$ (15 $\mathrm{mL}$ ). After stirring at room temperature for $3 \mathrm{~h}$, the solvent was removed under vacuum and the residue was dissolved in freshly distilled $\mathrm{CH}_{2} \mathrm{Cl}_{2}$ (50 mL). 8-Aminoquinoline (1.44 g, $10 \mathrm{mmol}$, 1 equiv) was added and the reaction was followed by TLC until it was completed. Water (100 $\mathrm{mL})$ was added. The aqueous layers were extracted with $\mathrm{CH}_{2} \mathrm{Cl}_{2}(3 \times 150 \mathrm{~mL})$. Then, the 
combined organic layers were dried over $\mathrm{MgSO}_{4}$. Removal of the solvent under vacuum and purification of the residue by Biotage afforded the corresponding amide.

The resulting amide derived from 8 -aminoquinoline ( $5 \mathrm{mmol}, 1.0$ equiv), $(\mathrm{CHO})_{\mathrm{n}}(0.44 \mathrm{~g}, 15$ mmol, 3.0 equiv), $(n \mathrm{Bu})_{4} \mathrm{NHSO}_{4}\left(0.17 \mathrm{~g}, 0.5 \mathrm{mmol}, 0.1\right.$ equiv) and $\mathrm{K}_{2} \mathrm{CO}_{3}(1.04 \mathrm{~g}, 7.5 \mathrm{mmol}$, 1.5 equiv) were added in toluene $(80 \mathrm{~mL})$ and stirred at $80{ }^{\circ} \mathrm{C}$. The reaction was followed by TLC until it was completed. Water $(100 \mathrm{~mL})$ was added. The aqueous layers were extracted with $\mathrm{Et}_{2} \mathrm{O}(3 \times 150 \mathrm{~mL})$. Then, the combined organic layers were dried over $\mathrm{Na}_{2} \mathrm{SO}_{4}$. Removal of the solvent under vacuum and purification of the residue by Biotage afforded the desired 2aryl- $N$-(quinolin-8-yl)acrylamides $\mathbf{1}$.

General procedure for the synthesis of 2-Phenyl-N-(quinolin-8-yl)acrylamide $1 \mathrm{f}$, $1 \mathrm{~s}$ and $\mathbf{1 t}$. Oxalyl chloride $\left(0.47 \mathrm{~mL}, 5.5 \mathrm{mmol}, 1.1\right.$ equiv) was slowly added at $0{ }^{\circ} \mathrm{C}$ to a solution of the corresponding acid (5 mmol, 1.0 equiv) and DMF (3 drops) in freshly distilled $\mathrm{CH}_{2} \mathrm{Cl}_{2}(40 \mathrm{~mL})$. After stirring at room temperature for $3 \mathrm{~h}$, the solvent was removed under vacuum and the residue was dissolved in freshly distilled $\mathrm{CH}_{2} \mathrm{Cl}_{2}(50 \mathrm{~mL})$. 8-Aminoquinoline $(0.72 \mathrm{~g}, 5 \mathrm{mmol}$, 1 equiv) was added and the reaction was followed by TLC until it was completed. Water (100 $\mathrm{mL})$ was added. The aqueous layers were extracted with $\mathrm{Et}_{2} \mathrm{O}(3 \times 150 \mathrm{~mL})$. Then, the combined organic layers were dried over $\mathrm{Na}_{2} \mathrm{SO}_{4}$. Removal of the solvent under vacuum and purification of the residue by Biotage afforded the desired product $\mathbf{1}$.

$N$-(5-Methoxyquinolin-8-yl)-2-phenylacrylamide 1u was prepared by using a similar procedure as the one depicted above for $\mathbf{1 f}$ by replacing the 8 -aminoquinoline with the 5 -methoxy-8aminoquinoline.

General procedure for the synthesis of $N$-methyl-N-(quinolin-8-yl)-2-(o-tolyl)acrylamide $\mathbf{1} \mathbf{w}^{18}$ To a suspension of sodium hydride (60\%) (80 mg, $2 \mathrm{mmol}, 2.0$ equiv) in dry DMF (5 mL), was added a solution of $\mathbf{1 m}\left(288 \mathrm{mg}, 1 \mathrm{mmol}, 1\right.$ equiv) in dry DMF $(10 \mathrm{~mL})$ at $0{ }^{\circ} \mathrm{C}$. Then, the reaction mixture was warmed to room temperature and stirred for $0.5 \mathrm{~h}$. Then, methyl iodide $\left(81 \mu \mathrm{L}, 1.3 \mathrm{mmol}, 1.3\right.$ equiv) was added dropwise and the reaction was stirred at $25^{\circ} \mathrm{C}$ for $3 \mathrm{~h}$. The reaction was diluted with $\mathrm{CH}_{2} \mathrm{Cl}_{2}(30 \mathrm{~mL})$ and the organic layer was washed with water (3 x $15 \mathrm{~mL}$ ), dried over $\mathrm{Na}_{2} \mathrm{SO}_{4}$. Removal of the solvent under vacuum and purification of the residue by Biotage afforded the desired product $\mathbf{1} \mathbf{w}$.

\section{Purification and Characterization of the Starting Materials 1.}

2-(4-(Hydroxymethyl)phenyl)-N-(quinolin-8-yl)-acrylamide (1d): Purification by silica gel column chromatography (Biotage system $25 \mathrm{~g}$, height $80 \mathrm{~mm}$, width $30 \mathrm{~mm}$, eluent: petroleum ether/diethyl ether, from 100/0 to 50/50). Yield: $45 \%$ (684 mg). $\mathrm{R}_{f}$ (petroleum ether/diethyl ether, 50/50): 0.41. White solid; m.p.: 96-97 ${ }^{\circ} \mathrm{C} .{ }^{1} \mathrm{H} \mathrm{NMR}\left(300.1 \mathrm{MHz}, \mathrm{CDCl}_{3}\right): \delta 10.25$ (brs, $1 \mathrm{H}), 8.87(\mathrm{~d}, J=7.2 \mathrm{~Hz}, 1 \mathrm{H}), 8.72-8.59(\mathrm{~m}, 1 \mathrm{H}), 8.11(\mathrm{~d}, J=8.1 \mathrm{~Hz}, 1 \mathrm{H}), 7.63-7.31(\mathrm{~m}, 7 \mathrm{H})$, $6.27(\mathrm{~s}, 1 \mathrm{H}), 5.82(\mathrm{~s}, 1 \mathrm{H}), 4.74(\mathrm{~s}, 2 \mathrm{H})$. Note that the proton of $\mathrm{OH}$ was not visible. ${ }^{13} \mathrm{C}\left\{{ }^{1} \mathrm{H}\right\}$ NMR $\left(75.5 \mathrm{MHz}, \mathrm{CDCl}_{3}\right): \delta 165.9,148.3,145.6,141.4,138.6,136.2,135.9,134.4,128.4$, 127.9, 127.3, 127.1, 121.9, 121.9, 121.5, 116.7, 64.9. IR (neat, $\mathrm{cm}^{-1}$ ) v: $3455,3350,2855,1670$, 1522, 1485, 1426, 1389, 1166, 1031, 826, 667. HRMS $\left(\mathrm{ESI}^{+}\right)$: calcd for $\mathrm{C}_{19} \mathrm{H}_{17} \mathrm{~N}_{2} \mathrm{O}_{2} \mathrm{~m} / \mathrm{z}$ $305.1290[\mathrm{M}+\mathrm{H}]^{+}$, found $305.1277(-4.3 \mathrm{ppm})$. 
2-(2-Bromophenyl)- $N$-(quinolin-8-yl)-acrylamide (10): Purification by silica gel column chromatography (Biotage system $25 \mathrm{~g}$, height $80 \mathrm{~mm}$, width $30 \mathrm{~mm}$, eluent: petroleum ether/diethyl ether, from 100/0 to 75/25). Yield: 66\% (1.18 g). $\mathrm{R}_{f}$ (petroleum ether/diethyl ether, 50/50): 0.52. Red solid; m.p.: 123-124 ${ }^{\circ} \mathrm{C} .{ }^{1} \mathrm{H}$ NMR (300.1 MHz, $\mathrm{CDCl}_{3}$ ): $\delta 9.90$ (brs, $\left.1 \mathrm{H}\right), 8.69$ $(\mathrm{dd}, J=7.5 \mathrm{~Hz}, 1.2 \mathrm{~Hz}, 1 \mathrm{H}), 8.37-8.32(\mathrm{~m}, 1 \mathrm{H}), 7.90-7.82(\mathrm{~m}, 1 \mathrm{H}), 7.49(\mathrm{~d}, J=7.8,1 \mathrm{H}), 7.36-$ $7.23(\mathrm{~m}, 4 \mathrm{H}), 7.17-7.09(\mathrm{~m}, 2 \mathrm{H}), 6.44(\mathrm{~d}, J=1.2 \mathrm{~Hz}, 1 \mathrm{H}), 5.55(\mathrm{~d}, J=0.9 \mathrm{~Hz}, 1 \mathrm{H}) .{ }^{13} \mathrm{C}\left\{{ }^{1} \mathrm{H}\right\}$ NMR (75.5 MHz, $\mathrm{CDCl}_{3}$ ): $\delta 163.7,148.2,145.3,138.6,138.3,136.1,134.4,133.0,131.7$, 130.1, 127.8, 127.8, 127.3, 125.7, 123.8, 121.7, 121.5, 116.4. IR (neat, $\mathrm{cm}^{-1}$ ) v: 3328, 1675, 1523, 1484, 1385, 1325, 1027, 790, 762, 682. HRMS $\left(\mathrm{ESI}^{+}\right)$: calcd for $\mathrm{C}_{18} \mathrm{H}_{14} \mathrm{BrN}_{2} \mathrm{O} \mathrm{m} / \mathrm{z}$ $353.0290[\mathrm{M}+\mathrm{H}]^{+}$, found $353.0296(1.7 \mathrm{ppm})$.

N-Methyl-N-(quinolin-8-yl)-2-(o-tolyl)-acrylamide (1w): Purification by silica gel column chromatography (Biotage system $25 \mathrm{~g}$, height $80 \mathrm{~mm}$, width $30 \mathrm{~mm}$, eluent: petroleum ether/diethyl ether, from 100/0 to 75/25). Yield: $70 \%$ (211 mg). $\mathrm{R}_{f}$ (petroleum ether/diethyl ether, 50/50): 0.59. Yellow oil. ${ }^{1} \mathrm{H}$ NMR (300.1 MHz, $\left.\mathrm{CDCl}_{3}\right): \delta 8.87-8.73$ (m, 1H), 7.99 (dd, $J$ $=8.4 \mathrm{~Hz}, 1.5 \mathrm{~Hz}, 1 \mathrm{H}), 7.67-7.53(\mathrm{~m}, 1 \mathrm{H}), 7.38-7.16(\mathrm{~m}, 3 \mathrm{H}), 6.66-6.50(\mathrm{~m}, 2 \mathrm{H}), 6.57-6.45(\mathrm{~m}$, $1 \mathrm{H}), 6.33-6.18(\mathrm{~m}, 1 \mathrm{H}), 6.07(\mathrm{~s}, 1 \mathrm{H}), 5.22(\mathrm{~s}, 1 \mathrm{H}), 3.54(\mathrm{~s}, 3 \mathrm{H}), 2.11(\mathrm{~s}, 3 \mathrm{H}) .{ }^{13} \mathrm{C}\left\{{ }^{1} \mathrm{H}\right\} \mathrm{NMR}$ (75.5 MHz, $\left.\mathrm{CDCl}_{3}\right): \delta 171.2,149.7,146.9,143.7,140.4,136.8,135.4,135.1,129.0,128.6$, 128.6, 128.1, 127.7, 126.3, 125.7, 124.6, 123.5, 121.2, 37.8, 19.7. IR (neat, $\mathrm{cm}^{-1}$ ) v: 2965, 2932, $1639,1613,1391,1354,1281,1197,1086,945,834,796$. HRMS $\left(\mathrm{ESI}^{+}\right)$: calcd for $\mathrm{C}_{20} \mathrm{H}_{19} \mathrm{~N}_{2} \mathrm{O}$ $\mathrm{m} / \mathrm{z} 303.1497[\mathrm{M}+\mathrm{H}]^{+}$, found $303.1501(1.3 \mathrm{ppm})$.

\section{General Procedure for the Synthesis of Derivatives 2.}

A dried tube was loaded with $\mathrm{PdCl}_{2}(7.1 \mathrm{mg}, 0.04 \mathrm{mmol}, 20 \mathrm{~mol} \%)$, reagent I (122.4 mg, 0.6 mmol, 3.0 equiv) and amide 1 ( $0.2 \mathrm{mmol}, 1.0$ equiv). Then DMF $(2 \mathrm{~mL})$ was injected. The tube was sealed with a cap and the suspension was stirred at $100{ }^{\circ} \mathrm{C}$ (oil bath) for $16 \mathrm{~h}$. After cooling down, the reaction was diluted with $\mathrm{Et}_{2} \mathrm{O}(30 \mathrm{~mL})$. Then, the organic layers were washed by water $(2 \times 10 \mathrm{~mL})$, by an aqueous saturated $\mathrm{NaHCO}_{3}$ solution $(10 \mathrm{~mL})$, dried over $\mathrm{Na}_{2} \mathrm{SO}_{4}$, and solvents were removed under vacuum. The residue was directly purified by silica gel column chromatography to give the desired product 2 . Note that for some compounds (see Scheme 2 in the manuscript), $10 \mathrm{~mol} \% \mathrm{PdCl}_{2}$ were used ( $3.5 \mathrm{mg}, 0.02 \mathrm{mmol}$ ).

\section{Purification and Characterization of the Isothiazolones 2.}

2-(Quinolin-8-yl)-4-(4-(tert-butyl)phenyl)-isothiazol-3(2H)-one (2a): Purification by silica gel column chromatography (height $18 \mathrm{~cm}$, width $1.5 \mathrm{~cm}$, eluent: $n$-pentane/ethyl acetate, from 100/0 to $75 / 25$ to $50 / 50$ ). Yield: $70 \%$ (50 mg). $\mathrm{R}_{f}$ ( $n$-pentane/ethyl acetate, 50/50): 0.39 . White solid; m.p.: 244-245 ${ }^{\circ} \mathrm{C} .{ }^{1} \mathrm{H}$ NMR $\left(300.1 \mathrm{MHz}, \mathrm{CDCl}_{3}\right): \delta 9.00-8.93(\mathrm{~m}, 1 \mathrm{H}), 8.43(\mathrm{~s}, 1 \mathrm{H}), 8.23$ $(\mathrm{d}, J=7.5 \mathrm{~Hz}, 1 \mathrm{H}), 8.05(\mathrm{~d}, J=7.2 \mathrm{~Hz}, 1 \mathrm{H}), 7.94-7.84(\mathrm{~m}, 3 \mathrm{H}), 7.68-7.59(\mathrm{~m}, 1 \mathrm{H}), 7.51-7.42$ (m, 3H), 1.35 (s, 9H). ${ }^{13} \mathrm{C}\left\{{ }^{1} \mathrm{H}\right\}$ NMR (75.5 MHz, $\left.\mathrm{CDCl}_{3}\right): \delta 167.3,151.0,150.6,143.9,136.4$, 135.2, 134.2, 133.7, 129.8, 129.6, 129.3, 128.9, 126.5, 126.3, 125.5, 123.7, 123.5, 121.8, 34.6, 31.3. IR (neat, $\mathrm{cm}^{-1}$ ) v: 2960, 1623, 1609, 1500, 1471, 1315, 1267, 831, 786. HRMS (ESI ${ }^{+}$): calcd for $\mathrm{C}_{22} \mathrm{H}_{21} \mathrm{~N}_{2} \mathrm{OS} \mathrm{m} / \mathrm{z} 361.1375[\mathrm{M}+\mathrm{H}]^{+}$, found 361.1388 (3.6 ppm). 
2-(Quinolin-8-yl)-4-(4-methylphenyl)-isothiazol-3(2H)-one (2b): Purification by silica gel column chromatography (height $18 \mathrm{~cm}$, width $1.5 \mathrm{~cm}$, eluent: $n$-pentane/ethyl acetate, from 100/0 to 75/25 to 50/50). Yield: $59 \%$ (38 mg). $\mathrm{R}_{f}$ (n-pentane/ethyl acetate, 50/50): 0.37. White solid; m.p.: 206-207 ${ }^{\circ} \mathrm{C} .{ }^{1} \mathrm{H}$ NMR $\left(300.1 \mathrm{MHz}, \mathrm{CDCl}_{3}\right): \delta 8.96(\mathrm{dd}, J=4.2 \mathrm{~Hz}, 1.8 \mathrm{~Hz}, 1 \mathrm{H})$, $8.42(\mathrm{~s}, 1 \mathrm{H}), 8.22(\mathrm{dd}, J=8.4 \mathrm{~Hz}, 1.8 \mathrm{~Hz}, 1 \mathrm{H}), 8.04(\mathrm{dd}, J=7.2 \mathrm{~Hz}, 1.2 \mathrm{~Hz}, 1 \mathrm{H}), 7.93-7.83$ (m, $3 \mathrm{H}), 7.67-7.60(\mathrm{~m}, 1 \mathrm{H}), 7.50-7.41(\mathrm{~m}, 1 \mathrm{H}), 7.25-7.20(\mathrm{~m}, 2 \mathrm{H}), 2.38(\mathrm{~s}, 3 \mathrm{H}) .{ }^{13} \mathrm{C}\left\{{ }^{1} \mathrm{H}\right\} \mathrm{NMR}$ (75.5 MHz, $\left.\mathrm{CDCl}_{3}\right): \delta 167.2,150.6,143.9,137.8,136.4,135.0,133.7,129.8,129.6,129.3$, 129.2, 128.9, 126.6, 126.2, 123.6, 121.8, 21.3. IR (neat, $\mathrm{cm}^{-1}$ ) v: 1647, 1495, 1387, 1267, 1186, 828, 793, 615, 529. HRMS $\left(\mathrm{ESI}^{+}\right)$: calcd for $\mathrm{C}_{19} \mathrm{H}_{15} \mathrm{~N}_{2} \mathrm{OS} \mathrm{m} / z 319.0905[\mathrm{M}+\mathrm{H}]^{+}$, found 319.0902 (-0.9 ppm).

2-(Quinolin-8-yl)-4-(4-methoxyphenyl)-isothiazol-3(2H)-one (2c): Purification by silica gel column chromatography (height $18 \mathrm{~cm}$, width $1.5 \mathrm{~cm}$, eluent: $n$-pentane/ethyl acetate, from $100 / 0$ to $75 / 25$ to $50 / 50$ ). Yield: $52 \%$ (35 mg). $\mathrm{R}_{f}$ (n-pentane/ethyl acetate, 50/50): 0.30 . Yellow solid; m.p.: 142-143 ${ }^{\circ} \mathrm{C} .{ }^{1} \mathrm{H}$ NMR $\left(300.1 \mathrm{MHz}, \mathrm{CDCl}_{3}\right): \delta 9.01-8.92(\mathrm{~m}, 1 \mathrm{H}), 8.37(\mathrm{~s}, 1 \mathrm{H}), 8.22$ $(\mathrm{d}, J=8.1 \mathrm{~Hz}, 1 \mathrm{H}), 8.02(\mathrm{~d}, J=7.5 \mathrm{~Hz}, 1 \mathrm{H}), 7.98-7.84(\mathrm{~m}, 3 \mathrm{H}), 7.69-7.58(\mathrm{~m}, 1 \mathrm{H}), 7.50-7.40$ $(\mathrm{m}, 1 \mathrm{H}), 6.96(\mathrm{~d}, J=8.7 \mathrm{~Hz}, 2 \mathrm{H}) .3 .83(\mathrm{~s}, 3 \mathrm{H}) .{ }^{13} \mathrm{C}\left\{{ }^{1} \mathrm{H}\right\} \mathrm{NMR}\left(75.5 \mathrm{MHz}, \mathrm{CDCl}_{3}\right): \delta 167.2$, $159.4,150.7,144.0,136.3,134.0,133.8,129.7,129.3,128.9,128.0,126.2,125.4,123.3,121.8$, 113.9, 55.3. IR (neat, $\mathrm{cm}^{-1}$ ) v: 2932, 2839, 1642, 1544, 1498, 1387, 1274, 1252, 1178, 1030, 822, 795, 759, 577. HRMS $\left(\mathrm{ESI}^{+}\right)$: calcd for $\mathrm{C}_{19} \mathrm{H}_{15} \mathrm{~N}_{2} \mathrm{O}_{2} \mathrm{~S} \mathrm{~m} / z 335.0854[\mathrm{M}+\mathrm{H}]^{+}$, found 335.0866 (3.6 ppm).

2-(Quinolin-8-yl)-4-(4-(hydroxymethyl)phenyl)-isothiazol-3(2H)-one (2d): Purification by silica gel column chromatography (height $18 \mathrm{~cm}$, width $1.5 \mathrm{~cm}$, eluent: $n$-pentane/ethyl acetate, from 100/0 to 75/25 to 50/50). Yield: $41 \%$ (27 mg). $\mathrm{R}_{f}$ ( $n$-pentane/ethyl acetate, 50/50): 0.22. Yellow solid; m.p.: $176-177{ }^{\circ} \mathrm{C} .{ }^{1} \mathrm{H}$ NMR $\left(300.1 \mathrm{MHz}, \mathrm{CDCl}_{3}\right): \delta 9.01-8.93(\mathrm{~m}, 1 \mathrm{H}), 8.51(\mathrm{~s}$, $1 \mathrm{H}), 8.25(\mathrm{dd}, J=8.1 \mathrm{~Hz}, 1.8 \mathrm{~Hz}, 1 \mathrm{H}), 8.09-7.89(\mathrm{~m}, 4 \mathrm{H}), 7.72-7.59(\mathrm{~m}, 1 \mathrm{H}), 7.53-7.40$ (m, $3 \mathrm{H}), 4.62(\mathrm{~s}, 2 \mathrm{H})$. Note that, the proton of $\mathrm{OH}$ is not visible. ${ }^{13} \mathrm{C}\left\{{ }^{1} \mathrm{H}\right\} \mathrm{NMR}\left(75.5 \mathrm{MHz}, \mathrm{CDCl}_{3}\right)$ : $\delta$ 167.0, 150.7, 143.9, 137.0, 136.4, 136.2, 133.6, 132.7, 129.6, 129.3, 129.0, 128.8, 127.0, 126.2, 123.0, 121.9, 46.1. IR (neat, $\mathrm{cm}^{-1}$ ) v: 3053, 2921, 2850, 1628, 1599, 1497, 1471, 1320 , 1259, 1203, 1132, 822, 783, 662. HRMS $\left(\mathrm{ESI}^{+}\right)$: calcd for $\mathrm{C}_{19} \mathrm{H}_{15} \mathrm{~N}_{2} \mathrm{O}_{2} \mathrm{~S} m / z 335.0854[\mathrm{M}+\mathrm{H}]^{+}$, found 335.0864 (3.0 ppm).

2-(Quinolin-8-yl)-4-([1,1'-biphenyl]-4-yl)-isothiazol-3(2H)-one (2e): Purification by silica gel column chromatography (height $18 \mathrm{~cm}$, width $1.5 \mathrm{~cm}$, eluent: $n$-pentane/ethyl acetate, from 100/0 to $75 / 25$ to $50 / 50$ ). Yield: $50 \%$ (38 mg). $\mathrm{R}_{f}$ (n-pentane/ethyl acetate, 50/50): 0.33 . Yellow solid; m.p.: 166-167 ${ }^{\circ} \mathrm{C} .{ }^{1} \mathrm{H}$ NMR $\left(300.1 \mathrm{MHz}, \mathrm{CDCl}_{3}\right): \delta 9.02-8.93(\mathrm{~m}, 1 \mathrm{H}), 8.53(\mathrm{~s}, 1 \mathrm{H}), 8.24$ $(\mathrm{dd}, J=8.4 \mathrm{~Hz}, 1.5 \mathrm{~Hz}, 1 \mathrm{H}), 8.11-8.03(\mathrm{~m}, 3 \mathrm{H}), 7.92(\mathrm{~d}, J=7.2 \mathrm{~Hz}, 1 \mathrm{H}), 7.71-7.58(\mathrm{~m}, 5 \mathrm{H})$, 7.50-7.32 (m, 4H). ${ }^{13} \mathrm{C}\left\{{ }^{1} \mathrm{H}\right\} \mathrm{NMR}\left(75.5 \mathrm{MHz}, \mathrm{CDCl}_{3}\right): \delta 167.1,150.7,143.9,140.7,140.6$, 136.4, 135.7, 133.6, 131.6, 129.6, 129.3, 129.0, 128.7, 127.3, 127.2, 127.0, 126.2, 123.5, 123.2, 121.9. IR (neat, $\mathrm{cm}^{-1}$ ) v: 2921, 1732, 1637, 1495, 1385, 1260, 1081, 823, 794, 765, 606. HRMS $\left(\mathrm{ESI}^{+}\right.$): calcd for $\mathrm{C}_{24} \mathrm{H}_{17} \mathrm{~N}_{2} \mathrm{OS} \mathrm{m} / z, 381.1062[\mathrm{M}+\mathrm{H}]^{+}$, found 381.1055 (-1.8 ppm). 
2-(Quinolin-8-yl)-4-phenyl-isothiazol-3(2H)-one (2f): Purification by silica gel column chromatography (height $18 \mathrm{~cm}$, width $1.5 \mathrm{~cm}$, eluent: $n$-pentane/ethyl acetate, from 100/0 to 75/25 to 50/50). Yield: $62 \%$ (37 mg). $\mathrm{R}_{f}$ (n-pentane/ethyl acetate, 50/50): 0.42. Yellow solid; m.p.: $222-223{ }^{\circ} \mathrm{C} .{ }^{1} \mathrm{H}$ NMR $\left(300.1 \mathrm{MHz}, \mathrm{CDCl}_{3}\right): \delta 8.98-8.91(\mathrm{~m}, 1 \mathrm{H}), 8.47(\mathrm{~s}, 1 \mathrm{H}), 8.20(\mathrm{dd}$, $J=7.2 \mathrm{~Hz}, 1.2 \mathrm{~Hz}, 1 \mathrm{H}), 8.06-7.85(\mathrm{~m}, 4 \mathrm{H}), 7.66-7.58(\mathrm{~m}, 1 \mathrm{H}), 7.48-7.30(\mathrm{~m}, 4 \mathrm{H}) .{ }^{13} \mathrm{C}\left\{{ }^{1} \mathrm{H}\right\}$ NMR (75.5 MHz, $\left.\mathrm{CDCl}_{3}\right): \delta 166.6,150.8,150.5,147.5,143.8,136.9,136.5,136.5,133.1$, 129.6, 129.4, 127.6, 126.2, 124.1, 122.0, 119.4. IR (neat, $\mathrm{cm}^{-1}$ ) v: 3092, 1646, 1596, 1501, 1458, 1316, 1273, 1104, 823, 784, 613, 477. HRMS (ESI ${ }^{+}$): calcd for $\mathrm{C}_{18} \mathrm{H}_{13} \mathrm{~N}_{2} \mathrm{OS} m / z 305.0749$ $[\mathrm{M}+\mathrm{H}]^{+}$, found $305.0756(2.3 \mathrm{ppm})$.

2-(Quinolin-8-yl)-4-(4-fluorophenyl)-isothiazol-3(2H)-one (2g): Purification by silica gel column chromatography (height $18 \mathrm{~cm}$, width $1.5 \mathrm{~cm}$, eluent: $n$-pentane/ethyl acetate, from $100 / 0$ to $75 / 25$ to $66 / 34$ ). Yield: $71 \%$ (46 mg), 65\% (209 mg) on $1 \mathrm{mmol}$ scale. $\mathrm{R}_{f}(n-$ pentane/ethyl acetate, 50/50): 0.43. Yellow solid; m.p.: 209-210 ${ }^{\circ} \mathrm{C} .{ }^{1} \mathrm{H}$ NMR $(300.1 \mathrm{MHz}$, $\left.\mathrm{CDCl}_{3}\right): \delta 9.00-8.93(\mathrm{~m}, 1 \mathrm{H}), 8.44(\mathrm{~s}, 1 \mathrm{H}), 8.24(\mathrm{~d}, J=8.1 \mathrm{~Hz}, 1 \mathrm{H}), 8.03(\mathrm{~d}, J=7.2 \mathrm{~Hz}, 1 \mathrm{H})$, 7.99-7.89 (m, 3H), 7.70-7.61 (m, 1H), 7.52-7.43 (m, 1H), 7.15-7.05 (m, 2H). ${ }^{19} \mathrm{~F}$ NMR $(282.4$ $\left.\mathrm{MHz}, \mathrm{CDCl}_{3}\right): \delta-113.9$ to $-114.2(\mathrm{~m}, 1 \mathrm{~F}) .{ }^{13} \mathrm{C}\left\{{ }^{1} \mathrm{H}\right\} \mathrm{NMR}\left(75.5 \mathrm{MHz}, \mathrm{CDCl}_{3}\right): \delta 167.0,162.5$ $(\mathrm{d}, J=247.6 \mathrm{~Hz}), 150.7,144.0,136.4,135.5,133.6,129.6,129.3,129.1,128.7$ (d, $J=3.0 \mathrm{~Hz})$, $128.5(\mathrm{~d}, J=8.3 \mathrm{~Hz}), 126.2,122.6,121.9,115.4$ (d, $J=21.1 \mathrm{~Hz})$. IR (neat, $\mathrm{cm}^{-1}$ ) v: 3064, 1635 , 1551, 1499, 1389, 1215, 1166, 810, 785, 561, 530. HRMS $\left(\mathrm{ESI}^{+}\right)$: calcd for $\mathrm{C}_{18} \mathrm{H}_{12} \mathrm{FN}_{2} \mathrm{OS} \mathrm{m} / \mathrm{z}$ $323.0654[\mathrm{M}+\mathrm{H}]^{+}$, found $323.0650(-1.2 \mathrm{ppm})$.

2-(Quinolin-8-yl)-4-(4-chlorophenyl)-isothiazol-3(2H)-one (2h): Purification by silica gel column chromatography (height $18 \mathrm{~cm}$, width $1.5 \mathrm{~cm}$, eluent: $n$-pentane/ethyl acetate, from $100 / 0$ to $75 / 25$ to $50 / 50$ ). Yield: $60 \%$ (41 mg). $\mathrm{R}_{f}$ ( $n$-pentane/ethyl acetate, 50/50): 0.40 . Yellow solid; m.p.: 208-209 ${ }^{\circ} \mathrm{C} .{ }^{1} \mathrm{H}$ NMR (300.1 MHz, $\left.\mathrm{CDCl}_{3}\right): \delta 9.00-8.93(\mathrm{~m}, 1 \mathrm{H}), 8.49(\mathrm{~s}, 1 \mathrm{H}), 8.24$ $(\mathrm{d}, J=8.1 \mathrm{~Hz}, 1 \mathrm{H}), 8.11(\mathrm{~d}, J=7.5 \mathrm{~Hz}, 1 \mathrm{H}), 7.98-7.89(\mathrm{~m}, 3 \mathrm{H}), 7.70-7.61(\mathrm{~m}, 1 \mathrm{H}), 7.52-7.45$ (m, 1H), 7.42-7.36 (m, 2H). ${ }^{13} \mathrm{C}\left\{{ }^{1} \mathrm{H}\right\}$ NMR $\left(75.5 \mathrm{MHz}, \mathrm{CDCl}_{3}\right): \delta 166.9,150.7,143.9,136.4$, 136.0, 133.8, 133.5, 131.0, 129.6, 129.3, 129.1, 128.7, 127.9, 126.2, 122.4, 121.9. IR (neat, $\mathrm{cm}^{-}$ $\left.{ }^{1}\right)$ v: $3048,1721,1632,1538,1470,1388,1317,1279,1090,1012,811,786,611,523,474$. HRMS (ESI ${ }^{+}$): calcd for $\mathrm{C}_{18} \mathrm{H}_{12} \mathrm{ClN}_{2} \mathrm{OS} m / z 339.0359[\mathrm{M}+\mathrm{H}]^{+}$, found 339.0360 (0.3 ppm).

2-(Quinolin-8-yl)-4-(4-iodophenyl)-isothiazol-3(2H)-one (2i): Purification by silica gel column chromatography (height $18 \mathrm{~cm}$, width $1.5 \mathrm{~cm}$, eluent: $n$-pentane/ethyl acetate, from 100/0 to 75/25 to 50/50). Yield: $45 \%$ (39 mg). $\mathrm{R}_{f}$ (n-pentane/ethyl acetate, 50/50): 0.37. Yellow solid; m.p.: $215-216{ }^{\circ} \mathrm{C} .{ }^{1} \mathrm{H}$ NMR $\left(300.1 \mathrm{MHz}, \mathrm{CDCl}_{3}\right): \delta 9.00-8.92(\mathrm{~m}, 1 \mathrm{H}), 8.50(\mathrm{~s}, 1 \mathrm{H}), 8.23$ (dd, $J=8.4 \mathrm{~Hz}, 1.5 \mathrm{~Hz}, 1 \mathrm{H}), 8.02(\mathrm{dd}, J=7.2 \mathrm{~Hz}, 0.9 \mathrm{~Hz}, 1 \mathrm{H}), 7.92(\mathrm{~d}, J=8.1 \mathrm{~Hz}, 1 \mathrm{H}), 7.78-7.62$ $(\mathrm{m}, 5 \mathrm{H}), 7.51-7.43(\mathrm{~m}, 1 \mathrm{H}) .{ }^{13} \mathrm{C}\left\{{ }^{1} \mathrm{H}\right\} \mathrm{NMR}\left(75.5 \mathrm{MHz}, \mathrm{CDCl}_{3}\right): \delta 166.8,150.7,143.9,137.6$, 136.4, 136.1, 133.4, 132.0, 129.6, 129.3, 129.1, 128.3, 126.2, 122.4, 121.9, 93.7. IR (neat, $\mathrm{cm}^{-}$ $\left.{ }^{1}\right)$ v: 3044, 2162, 1630, 1532, 1470, 1388, 1321, 1198, 1004, 827, 799, 609, 523. HRMS (ESI $\left.{ }^{+}\right)$: calcd for $\mathrm{C}_{18} \mathrm{H}_{12} \mathrm{IN}_{2} \mathrm{OS} \mathrm{m} / z 430.9715[\mathrm{M}+\mathrm{H}]^{+}$, found 430.9708 (-1.6 ppm). 
2-(Quinolin-8-yl)-4-(3-methylphenyl)-isothiazol-3(2H)-one (2j): Purification by silica gel column chromatography (height $18 \mathrm{~cm}$, width $1.5 \mathrm{~cm}$, eluent: $n$-pentane/ethyl acetate, from $100 / 0$ to $75 / 25$ to $50 / 50$ ). Yield: $61 \%$ (39 mg). $\mathrm{R}_{f}$ (n-pentane/ethyl acetate, 50/50): 0.36 . Yellow solid; m.p.: $180-181{ }^{\circ} \mathrm{C} .{ }^{1} \mathrm{H}$ NMR $\left(300.1 \mathrm{MHz}, \mathrm{CDCl}_{3}\right)$ : $\delta 9.00-8.92(\mathrm{~m}, 1 \mathrm{H}), 8.46(\mathrm{~s}, 1 \mathrm{H}), 8.22$ $(\mathrm{dd}, J=8.4 \mathrm{~Hz}, 1.2 \mathrm{~Hz}, 1 \mathrm{H}), 8.03(\mathrm{~d}, J=7.5 \mathrm{~Hz}, 1 \mathrm{H}), 7.89$ (d, $J=8.1 \mathrm{~Hz}, 1 \mathrm{H}), 7.83(\mathrm{~s}, 1 \mathrm{H})$, $7.72(\mathrm{~d}, J=7.8 \mathrm{~Hz}, 1 \mathrm{H}), 7.67-7.58(\mathrm{~m}, 1 \mathrm{H}), 7.50-7.41(\mathrm{~m}, 1 \mathrm{H}), 7.36-7.27(\mathrm{~m}, 1 \mathrm{H}), 7.20-7.12$ (m, 1H), 2.39 (s, 3H). ${ }^{13} \mathrm{C}\left\{{ }^{1} \mathrm{H}\right\}$ NMR (75.5 MHz, $\left.\mathrm{CDCl}_{3}\right): \delta 167.1,150.6,143.9,138.0,136.3$, $135.8,133.7,132.5,129.5,129.2$, 128.9, 128.7, 128.3, 127.3, 126.1, 123.8, 123.6, 121.8, 21.5. IR (neat, $\mathrm{cm}^{-1}$ ) v: 3048, 1644, 1496, 1380, 1324, 1278, 1149, 830, 787, 693, 608. HRMS (ESI ${ }^{+}$): calcd for $\mathrm{C}_{19} \mathrm{H}_{15} \mathrm{~N}_{2} \mathrm{OS} \mathrm{m} / z 319.0905[\mathrm{M}+\mathrm{H}]^{+}$, found 319.0913 (2.5 ppm).

2-(Quinolin-8-yl)-4-(3-chlorophenyl)-isothiazol-3(2H)-one (2k): Purification by silica gel column chromatography (height $18 \mathrm{~cm}$, width $1.5 \mathrm{~cm}$, eluent: $n$-pentane/ethyl acetate, from 100/0 to $75 / 25$ to $50 / 50$ ). Yield: $51 \%$ (35 mg). $\mathrm{R}_{f}$ (n-pentane/ethyl acetate, 50/50): 0.39. Yellow solid; m.p.: 150-151 ${ }^{\circ} \mathrm{C} .{ }^{1} \mathrm{H}$ NMR $\left(300.1 \mathrm{MHz}, \mathrm{CDCl}_{3}\right): \delta 9.00-8.91(\mathrm{~m}, 1 \mathrm{H}), 8.51(\mathrm{~s}, 1 \mathrm{H}), 8.23$ $(\mathrm{d}, J=8.1 \mathrm{~Hz}, 1 \mathrm{H}), 8.06-7.98(\mathrm{~m}, 2 \mathrm{H}), 7.94-7.82(\mathrm{~m}, 2 \mathrm{H}), 7.70-7.59(\mathrm{~m}, 1 \mathrm{H}), 7.50-7.43(\mathrm{~m}$, 1H), 7.38-7.28 (m, 2H). ${ }^{13} \mathrm{C}\left\{{ }^{1} \mathrm{H}\right\}$ NMR $\left(75.5 \mathrm{MHz}, \mathrm{CDCl}_{3}\right): \delta 166.8,150.7,143.9,136.7,136.4$, $134.5,134.2,133.4,129.7,129.6,129.3,129.1,128.0,126.6,126.2,124.7,122.1,121.9$. IR (neat, $\mathrm{cm}^{-1}$ ) v: 3064, 1727, 1651, 1596, 1502, 1472, 1319, 1280, 1149, 825, 778, 608. HRMS $\left(\mathrm{ESI}^{+}\right.$): calcd for $\mathrm{C}_{18} \mathrm{H}_{12} \mathrm{ClN}_{2} \mathrm{OS} \mathrm{m} / z 339.0359[\mathrm{M}+\mathrm{H}]^{+}$, found 339.0364 (1.5 ppm).

2-(Quinolin-8-yl)-4-(3-(trifluoromethyl)phenyl)-isothiazol-3(2H)-one (2I): Purification by silica gel column chromatography (height $18 \mathrm{~cm}$, width $1.5 \mathrm{~cm}$, eluent: $n$-pentane/ethyl acetate, from 100/0 to $75 / 25$ to 50/50). Yield: $65 \%$ (48 mg). $\mathrm{R}_{f}$ ( $n$-pentane/ethyl acetate, 50/50): 0.34 . Yellow solid; m.p.: $140-141{ }^{\circ} \mathrm{C} .{ }^{1} \mathrm{H}$ NMR (300.1 MHz, $\left.\mathrm{CDCl}_{3}\right): \delta 9.01-8.89(\mathrm{~m}, 1 \mathrm{H}), 8.58$ (s, $1 \mathrm{H}), 8.33-8.10(\mathrm{~m}, 3 \mathrm{H}), 8.06-7.98(\mathrm{~m}, 1 \mathrm{H}), 7.91(\mathrm{~d}, J=8.1 \mathrm{~Hz}, 1 \mathrm{H}), 7.69-7.43(\mathrm{~m}, 4 \mathrm{H}) .{ }^{19} \mathrm{~F}$ NMR (282.4 MHz, $\left.\mathrm{CDCl}_{3}\right): \delta-63.1(\mathrm{~s}, 3 \mathrm{~F}) .{ }^{13} \mathrm{C}\left\{{ }^{1} \mathrm{H}\right\} \mathrm{NMR}\left(75.5 \mathrm{MHz}, \mathrm{CDCl}_{3}\right): \delta 166.8,150.7$, 143.9, 137.1, 136.4, 133.3, 133.2, 130.1 (q, $J=32.5 \mathrm{~Hz}), 129.7$ (q, $J=1.1 \mathrm{~Hz}), 129.6,129.3$, 129.2, 128.9, 126.1, $124.4(\mathrm{q}, J=3.8 \mathrm{~Hz}), 124.1(\mathrm{q}, J=277.1 \mathrm{~Hz}), 123.3(\mathrm{q}, J=3.8 \mathrm{~Hz}), 121.9$. IR (neat, $\mathrm{cm}^{-1}$ ) v: $3053,1651,1503,1473,1391,1331,1262,1114,1072,826,789$. HRMS $\left(\mathrm{ESI}^{+}\right)$: calcd for $\mathrm{C}_{19} \mathrm{H}_{12} \mathrm{~F}_{3} \mathrm{~N}_{2} \mathrm{OS} \mathrm{m} / z$ 373.0622 [M+H] $]^{+}$, found 373.0612 (-2.7 ppm).

2-(Quinolin-8-yl)-4-(2-methylphenyl)-isothiazol-3(2H)-one (2m): Purification by silica gel column chromatography (height $18 \mathrm{~cm}$, width $1.5 \mathrm{~cm}$, eluent: $n$-pentane/ethyl acetate, from 100/0 to $75 / 25$ to $50 / 50$ ). Yield: $68 \%$ (43 mg). $\mathrm{R}_{f}$ ( $n$-pentane/ethyl acetate, 50/50): 0.36 . White solid; m.p.: 156-157 ${ }^{\circ} \mathrm{C} .{ }^{1} \mathrm{H}$ NMR $\left(300.1 \mathrm{MHz}, \mathrm{CDCl}_{3}\right)$ : $\delta$ 8.98-8.93 (m, 1H), 8.27-8.16 (m, $2 \mathrm{H}), 8.09(\mathrm{~d}, J=7.5 \mathrm{~Hz}, 1 \mathrm{H}), 7.85(\mathrm{~d}, J=8.1 \mathrm{~Hz}, 1 \mathrm{H}), 7.66-7.57(\mathrm{~m}, 1 \mathrm{H}), 7.49-7.40(\mathrm{~m}, 2 \mathrm{H})$, 7.30-7.19 (m, 3H), $2.44(\mathrm{~s}, 3 \mathrm{H}) .{ }^{13} \mathrm{C}\left\{{ }^{1} \mathrm{H}\right\}$ NMR (75.5 MHz, $\left.\mathrm{CDCl}_{3}\right): \delta 167.2,150.3,143.6$, 138.2, 137.3, 136.3, 133.9, 132.6, 130.4, 130.2, 129.2, 129.0, 128.5, 128.3, 126.2, 125.8, 125.7, 121.7, 20.3. IR (neat, $\mathrm{cm}^{-1}$ ) v: 3064, 2927, 1651, 1592, 1501, 1472, 1312, 1130, 829, 744, 606, 452. HRMS (ESI ${ }^{+}$): calcd for $\mathrm{C}_{19} \mathrm{H}_{15} \mathrm{~N}_{2} \mathrm{OS} \mathrm{m} / z 319.0905[\mathrm{M}+\mathrm{H}]^{+}$, found 319.0901 (-1.3 ppm). 
2-(Quinolin-8-yl)-4-(2-chlorophenyl)-isothiazol-3(2H)-one (2n): Purification by silica gel column chromatography (height $18 \mathrm{~cm}$, width $1.5 \mathrm{~cm}$, eluent: $n$-pentane/ethyl acetate, from 100/0 to $75 / 25$ to $50 / 50$ ). Yield: $62 \%$ (42 $\mathrm{mg}$ ). $\mathrm{R}_{f}$ (n-pentane/ethyl acetate, 50/50): 0.41 . Yellow solid; m.p.: 84-85 ${ }^{\circ} \mathrm{C} .{ }^{1} \mathrm{H}$ NMR (300.1 MHz, $\left.\mathrm{CDCl}_{3}\right)$ : $\delta$ 9.01-8.94 (m, 1H), $8.67(\mathrm{~s}, 1 \mathrm{H}), 8.22$ $(\mathrm{dd}, J=8.1 \mathrm{~Hz}, 1.5 \mathrm{~Hz}, 1 \mathrm{H}), 8.11(\mathrm{dd}, J=7.5 \mathrm{~Hz}, 1.2 \mathrm{~Hz}, 1 \mathrm{H}), 7.88(\mathrm{dd}, J=8.1 \mathrm{~Hz}, 0.9 \mathrm{~Hz}$, $1 \mathrm{H}), 7.81(\mathrm{dd}, J=7.5 \mathrm{~Hz}, 2.1 \mathrm{~Hz}, 1 \mathrm{H}), 7.67-7.58(\mathrm{~m}, 1 \mathrm{H}), 7.52-7.44(\mathrm{~m}, 2 \mathrm{H}), 7.36-7.24(\mathrm{~m}$, $2 \mathrm{H}) .{ }^{13} \mathrm{C}\left\{{ }^{1} \mathrm{H}\right\} \mathrm{NMR}\left(75.5 \mathrm{MHz}, \mathrm{CDCl}_{3}\right): \delta 167.1,150.4,143.5,140.5,136.4,133.6,132.9$, 131.3, 131.2, 130.0, 129.2, 129.1, 129.1, 128.6, 126.7, 126.2, 121.8, 121.2. IR (neat, $\mathrm{cm}^{-1}$ ) v: 3053, 2233, 1628, 1498, 1468, 1387, 1320, 907, 823, 723, 564. HRMS (ESI ${ }^{+}$): calcd for $\mathrm{C}_{18} \mathrm{H}_{12} \mathrm{ClN}_{2} \mathrm{OS} \mathrm{m} / z 339.0359[\mathrm{M}+\mathrm{H}]^{+}$, found 339.0374 (4.4 ppm).

2-(Quinolin-8-yl)-4-(2-bromophenyl)-isothiazol-3(2H)-one (2o): Purification by silica gel column chromatography (height $18 \mathrm{~cm}$, width $1.5 \mathrm{~cm}$, eluent: $n$-pentane/ethyl acetate, from $100 / 0$ to $75 / 25$ to $50 / 50$ ). Yield: $65 \%$ (50 mg). $\mathrm{R}_{f}$ ( $n$-pentane/ethyl acetate, $\left.50 / 50\right): 0.38$. Yellow solid. m.p.: 64-65 ${ }^{\circ} \mathrm{C} .{ }^{1} \mathrm{H}$ NMR (300.1 MHz, $\left.\mathrm{CDCl}_{3}\right): \delta 9.03-8.93(\mathrm{~m}, 1 \mathrm{H}), 8.62(\mathrm{~s}, 1 \mathrm{H}), 8.22$ $(\mathrm{dd}, J=8.4 \mathrm{~Hz}, 1.5 \mathrm{~Hz}, 1 \mathrm{H}), 8.12(\mathrm{dd}, J=7.8 \mathrm{~Hz}, 1.2 \mathrm{~Hz}, 1 \mathrm{H}), 7.87(\mathrm{~d}, J=8.4 \mathrm{~Hz}, 1 \mathrm{H}), 7.74-$ $7.59(\mathrm{~m}, 3 \mathrm{H}), 7.51-7.42(\mathrm{~m}, 1 \mathrm{H}), 7.41-7.32(\mathrm{~m}, 1 \mathrm{H}), 7.24-7.15(\mathrm{~m}, 1 \mathrm{H}) .{ }^{13} \mathrm{C}\left\{{ }^{1} \mathrm{H}\right\}$ NMR $(75.5$ $\left.\mathrm{MHz}, \mathrm{CDCl}_{3}\right): \delta 167.0,150.3,143.5,140.5,136.4,133.6,133.3,133.2,131.6,129.4,129.2$, 129.0, 128.5, 127.3, 126.2, 123.2, 123.1, 121.8. IR (neat, $\mathrm{cm}^{-1}$ ) v: 2927, 2233, 1721, 1629, 1595, 1498, 1469, 1320, 1283, 823, 723, 607. HRMS (ESI ${ }^{+}$: calcd for $\mathrm{C}_{18} \mathrm{H}_{12} \mathrm{BrN}_{2} \mathrm{OS} \mathrm{m} / \mathrm{z}$ $384.9833[\mathrm{M}+\mathrm{H}]^{+}$, found $384.9840(1.8 \mathrm{ppm})$.

2-(Quinolin-8-yl)-4-(3,4-dimethoxyphenyl)-isothiazol-3(2H)-one (2p): Purification by silica gel column chromatography (height $18 \mathrm{~cm}$, width $1.5 \mathrm{~cm}$, eluent: $n$-pentane/ethyl acetate, from 100/0 to $50 / 50$ to $34 / 66$ ). Yield: $47 \%$ (34 mg). $\mathrm{R}_{f}$ (n-pentane/ethyl acetate, 50/50): 0.15. Yellow solid; m.p.: 209-210 ${ }^{\circ} \mathrm{C} .{ }^{1} \mathrm{H}$ NMR $\left(300.1 \mathrm{MHz}, \mathrm{CDCl}_{3}\right): \delta 9.02-8.91(\mathrm{~m}, 1 \mathrm{H}), 8.42(\mathrm{~s}, 1 \mathrm{H}), 8.25$ $(\mathrm{d}, J=8.4 \mathrm{~Hz}, 1 \mathrm{H}), 8.02(\mathrm{~d}, J=7.5 \mathrm{~Hz}, 1 \mathrm{H}), 7.93(\mathrm{~d}, J=8.1 \mathrm{~Hz}, 1 \mathrm{H}), 7.82-7.75(\mathrm{~m}, 1 \mathrm{H}), 7.71-$ $7.61(\mathrm{~m}, 1 \mathrm{H}), 7.54-7.39(\mathrm{~m}, 2 \mathrm{H}), 6.93(\mathrm{~d}, J=8.4 \mathrm{~Hz}, 1 \mathrm{H}), 3.92(\mathrm{~s}, 6 \mathrm{H}) .{ }^{13} \mathrm{C}\left\{{ }^{1} \mathrm{H}\right\} \mathrm{NMR}(75.5$ $\left.\mathrm{MHz}, \mathrm{CDCl}_{3}\right): \delta 167.3,150.8,148.9,148.8,144.1,136.4,134.2,133.7,129.9,129.4,129.2$, $126.3,125.7,123.1,121.9,119.2,111.1,109.9,55.9,55.9$. IR (neat, $\mathrm{cm}^{-1}$ ) v: 2960, 2916, 1643, 1497, 1330, 1244, 1135, 1022, 795, 593. HRMS (ESI ${ }^{+}$): calcd for $\mathrm{C}_{20} \mathrm{H}_{17} \mathrm{~N}_{2} \mathrm{O}_{3} \mathrm{~S} \mathrm{~m} / z, 365.0960$ $[\mathrm{M}+\mathrm{H}]^{+}$, found $365.0964(1.1 \mathrm{ppm})$.

2-(Quinolin-8-yl)-4-(benzo[d][1,3]dioxol-5-yl)-isothiazol-3(2H)-one (2q): Purification by silica gel column chromatography (height $18 \mathrm{~cm}$, width $1.5 \mathrm{~cm}$, eluent: $n$-pentane/ethyl acetate, from 100/0 to 75/25 to 50/50). Yield: $48 \%$ (34 mg). $\mathrm{R}_{f}$ ( $n$-pentane/ethyl acetate, 50/50): 0.28. Yellow solid; m.p.: $234-235{ }^{\circ} \mathrm{C} .{ }^{1} \mathrm{H}$ NMR $\left(300.1 \mathrm{MHz}, \mathrm{CDCl}_{3}\right): \delta 9.03-8.91(\mathrm{~m}, 1 \mathrm{H}), 8.36$ (s, $1 \mathrm{H}), 8.24(\mathrm{~d}, J=8.1 \mathrm{~Hz}, 1 \mathrm{H}), 8.02(\mathrm{~d}, J=7.2 \mathrm{~Hz}, 1 \mathrm{H}), 7.91(\mathrm{~d}, J=8.1 \mathrm{~Hz}, 1 \mathrm{H}), 7.73-7.60(\mathrm{~m}$, $1 \mathrm{H}), 7.56-7.41(\mathrm{~m}, 3 \mathrm{H}), 6.87(\mathrm{~d}, J=8.4 \mathrm{~Hz}, 1 \mathrm{H}), 5.98(\mathrm{~s}, 2 \mathrm{H}) .{ }^{13} \mathrm{C}\left\{{ }^{1} \mathrm{H}\right\}$ NMR $(75.5 \mathrm{MHz}$, $\left.\mathrm{CDCl}_{3}\right): \delta 167.0,150.7,147.8,147.4,144.0,136.4,134.5,133.7,129.6,129.3,129.0,126.8$, 126.2, 123.2, 121.9, 120.6, 108.4, 107.3, 101.1. IR (neat, $\mathrm{cm}^{-1}$ ) v: 3097, 2921, 1628, 1490, 1388, 1323, 1257, 1108, 1032, 932, 821, 801, 768, 615. HRMS (ESI ${ }^{+}$): calcd for $\mathrm{C}_{19} \mathrm{H}_{13} \mathrm{~N}_{2} \mathrm{O}_{3} \mathrm{~S}$ $\mathrm{m} / \mathrm{z} 349.0647[\mathrm{M}+\mathrm{H}]^{+}$, found $349.0651(1.1 \mathrm{ppm})$. 
2-(Quinolin-8-yl)-4-(6-chloropyridin-3-yl)-isothiazol-3(2H)-one (2r): Purification by silica gel column chromatography (height $18 \mathrm{~cm}$, width $1.5 \mathrm{~cm}$, eluent: $n$-pentane/ethyl acetate, from 100/0 to 66/34 to 50/50). Yield: $45 \%$ (31 mg). $\mathrm{R}_{f}$ (n-pentane/ethyl acetate, 50/50): 0.22. Yellow solid; m.p.: $151-152{ }^{\circ} \mathrm{C} .{ }^{1} \mathrm{H}$ NMR $\left(300.1 \mathrm{MHz}, \mathrm{CDCl}_{3}\right)$ : $\delta$ 8.99-8.92 (m, 1H), 8.91-8.83 (m, $1 \mathrm{H}), 8.61(\mathrm{~s}, 1 \mathrm{H}), 8.40(\mathrm{dd}, J=8.1 \mathrm{~Hz}, 2.4 \mathrm{~Hz}, 1 \mathrm{H}), 8.25(\mathrm{dd}, J=8.4 \mathrm{~Hz}, 1.5 \mathrm{~Hz}, 1 \mathrm{H}), 8.02$ (d, $J=7.5 \mathrm{~Hz}, 1 \mathrm{H}), 7.94(\mathrm{~d}, J=8.1 \mathrm{~Hz}, 1 \mathrm{H}), 7.70-7.61(\mathrm{~m}, 1 \mathrm{H}), 7.54-7.45(\mathrm{~m}, 1 \mathrm{H}), 7.38(\mathrm{~d}, J=$ $8.4 \mathrm{~Hz}, 1 \mathrm{H}) .{ }^{13} \mathrm{C}\left\{{ }^{1} \mathrm{H}\right\} \mathrm{NMR}\left(75.5 \mathrm{MHz}, \mathrm{CDCl}_{3}\right): \delta 166.6,150.8,150.4,147.5,143.8,136.9$, 136.5, 136.4, 133.1, 129.6, 129.4, 129.3, 127.6, 126.2, 124.0, 122.0, 119.3. IR (neat, $\mathrm{cm}^{-1}$ ) v: 3053, 1651, 1500, 1470, 1318, 1145, 1276, 824, 762, 694, 605. HRMS (ESI ${ }^{+}$): calcd for $\mathrm{C}_{17} \mathrm{H}_{11} \mathrm{ClN}_{3} \mathrm{OS} \mathrm{m} / \mathrm{z} 340.0311[\mathrm{M}+\mathrm{H}]^{+}$, found 340.0308 (-0.9 ppm).

2-(Quinolin-8-yl)-4,5-diphenyl-isothiazol-3(2H)-one (2s): Purification by silica gel column chromatography (height $18 \mathrm{~cm}$, width $1.5 \mathrm{~cm}$, eluent: $n$-pentane/ethyl acetate, from 100/0 to 66/34 to 50/50). Yield: $49 \%$ (38 mg). $\mathrm{R}_{f}$ ( $n$-pentane/ethyl acetate, 50/50): 0.31. Yellow solid; m.p.: 229-230 ${ }^{\circ} \mathrm{C} .{ }^{1} \mathrm{H}$ NMR $\left(300.1 \mathrm{MHz}, \mathrm{CDCl}_{3}\right): \delta 9.05-8.96(\mathrm{~m}, 1 \mathrm{H}), 8.25(\mathrm{~d}, J=8.4 \mathrm{~Hz}, 1 \mathrm{H})$, $8.19(\mathrm{~d}, J=7.5 \mathrm{~Hz}, 1 \mathrm{H}), 7.89(\mathrm{~d}, J=8.1 \mathrm{~Hz}, 1 \mathrm{H}), 7.69-7.61(\mathrm{~m}, 1 \mathrm{H}), 7.52-7.45(\mathrm{~m}, 3 \mathrm{H}), 7.40-$ $7.27(\mathrm{~m}, 8 \mathrm{H}) .{ }^{13} \mathrm{C}\left\{{ }^{1} \mathrm{H}\right\} \mathrm{NMR}\left(75.5 \mathrm{MHz}, \mathrm{CDCl}_{3}\right): \delta 168.0,152.9,150.3,143.6,136.5,133.9$, 132.4 131.0, 130.1, 130.0, 129.3, 129.0, 128.9, 128.5, 128.3, 128.2, 127.7, 126.3, 121.8, 121.6. IR (neat, $\mathrm{cm}^{-1}$ ) v: 2921, 1652, 1502, 823, 779. 695, 601, 508. HRMS (ESI ${ }^{+}$: calcd for $\mathrm{C}_{24} \mathrm{H}_{17} \mathrm{~N}_{2} \mathrm{OS} \mathrm{m} / z 381.1062[\mathrm{M}+\mathrm{H}]^{+}$, found 381.1078 (4.2 ppm).

2-(Quinolin-8-yl)-4,5-dimethyl-isothiazol-3(2H)-one (2t): Purification by silica gel column chromatography (height $18 \mathrm{~cm}$, width $1.5 \mathrm{~cm}$, eluent: $n$-pentane/ethyl acetate, from 100/0 to 66/34 to 75/25). Yield: $51 \%$ (26 mg). $\mathrm{R}_{f}$ (n-pentane/ethyl acetate, 50/50): 0.12. Yellow solid; m.p.: $200-201{ }^{\circ} \mathrm{C} .{ }^{1} \mathrm{H}$ NMR $\left(300.1 \mathrm{MHz}, \mathrm{CDCl}_{3}\right): \delta 9.02-8.92(\mathrm{~m}, 1 \mathrm{H}), 8.22(\mathrm{dd}, J=8.4 \mathrm{~Hz}$, $1.5 \mathrm{~Hz}, 1 \mathrm{H}), 7.97$ (d, $J=7.2 \mathrm{~Hz}, 1 \mathrm{H}), 7.87(\mathrm{~d}, J=8.1 \mathrm{~Hz}, 1 \mathrm{H}), 7.67-7.56(\mathrm{~m}, 1 \mathrm{H}), 7.49-7.40$ (m, 1H), 2.40 (s, 3H), 2.07 (s, 3H). ${ }^{13} \mathrm{C}\left\{{ }^{1} \mathrm{H}\right\}$ NMR (75.5 MHz, $\left.\mathrm{CDCl}_{3}\right): \delta 169.4,150.4,147.4$, 144.1, 136.3, 134.1, 129.3, 129.3, 128.4, 126.3, 121.7, 118.6, 13.1, 10.7. IR (neat, $\mathrm{cm}^{-1}$ ) v: 2916, 1634, 1496, 1328, 829, 793. 695, 624, 509. HRMS (ESI $\left.{ }^{+}\right)$: calcd for $\mathrm{C}_{14} \mathrm{H}_{13} \mathrm{~N}_{2} \mathrm{OS} \mathrm{m} / \mathrm{z}$ $257.0749[\mathrm{M}+\mathrm{H}]^{+}$, found $257.0745(-1.6 \mathrm{ppm})$.

2-(5-Methoxyquinolin-8-yl)-4-phenyl-isothiazol-3(2H)-one (2u): Purification by silica gel column chromatography (height $18 \mathrm{~cm}$, width $1.5 \mathrm{~cm}$, eluent: $n$-pentane/ethyl acetate, from $100 / 0$ to $75 / 25$ to $50 / 50$ ). Yield: $50 \%$ (33 mg), $49 \%$ (82 mg) on $0.5 \mathrm{mmol}$ scale. $\mathrm{R}_{f}(n-$ pentane/ethyl acetate, 50/50): 0.40. Yellow solid; m.p.: 156-157 ${ }^{\circ} \mathrm{C} .{ }^{1} \mathrm{H}$ NMR $(300.1 \mathrm{MHz}$, $\left.\mathrm{CDCl}_{3}\right): \delta 9.03-8.84(\mathrm{~m}, 1 \mathrm{H}), 8.61(\mathrm{~d}, J=8.4 \mathrm{~Hz}, 1 \mathrm{H}), 8.45(\mathrm{~s}, 1 \mathrm{H}), 7.97(\mathrm{~d}, J=7.2 \mathrm{~Hz}, 2 \mathrm{H})$, $7.87(\mathrm{~d}, J=7.8 \mathrm{~Hz}, 1 \mathrm{H}), 7.50-7.30(\mathrm{~m}, 4 \mathrm{H}), 6.92(\mathrm{~d}, J=8.4 \mathrm{~Hz}, 1 \mathrm{H}), 4.03(\mathrm{~s}, 3 \mathrm{H}) .{ }^{13} \mathrm{C}\left\{{ }^{1} \mathrm{H}\right\}$ NMR (75.5 MHz, $\mathrm{CDCl}_{3}$ ): $\delta$ 167.2, 155.9, 151.1, 144.9, 135.4, 132.7, 131.2, 130.5, 128.5, 127.9, 126.7, 125.9, 123.7, 121.6, 120.9, 103.7, 56.0. IR (neat, $\mathrm{cm}^{-1}$ ) v: 3070, 1728, 1656, 1590, 1477, 1369, 1276, 1153, 1091, 811, 770, 695, 610. HRMS $\left(\mathrm{ESI}^{+}\right)$: calcd for $\mathrm{C}_{19} \mathrm{H}_{15} \mathrm{~N}_{2} \mathrm{O}_{2} \mathrm{~S} \mathrm{~m} / \mathrm{z}$ $335.0854[\mathrm{M}+\mathrm{H}]^{+}$, found $335.0864(3.0 \mathrm{ppm})$. 
Post-functionalization reaction of the product $2 \mathbf{u}^{\mathbf{1 8}} \mathrm{In}$ a dried tube was added $\mathbf{2 u}(33.4 \mathrm{mg}$, $0.1 \mathrm{mmol})$ and TCCA (23.2 mg, $0.1 \mathrm{mmol}, 1$ equiv) under air. Then acetonitrile ( $1 \mathrm{~mL})$ was injected. The tube was sealed with a cap and the suspension was stirred at room temperature for $6 \mathrm{~h}$. The reaction was diluted with acetonitrile $(15 \mathrm{~mL})$. Then, the organic layers were washed by water $(2 \times 10 \mathrm{~mL})$, by an aqueous saturated $\mathrm{NaHCO}_{3}$ solution $(10 \mathrm{~mL})$, dried over $\mathrm{Na}_{2} \mathrm{SO}_{4}$, and solvents were removed under vacuum. The residue was directly purified by silica gel column chromatography to afford the desired product $6(20 \mathrm{mg}, 55 \%)$.

$\mathrm{N}$-(7-Chloro-5-methoxyquinolin-8-yl)-4-phenyl-isothiazol-3(2H)-one (6): Purification by silica gel column chromatography (height $18 \mathrm{~cm}$, width $1.5 \mathrm{~cm}$, eluent: $n$-pentane/ethyl acetate, from 100/0 to 75/25). Yield: $55 \%$ (20 mg). $\mathrm{R}_{f}$ (n-pentane/ethyl acetate, 50/50): 0.65. Yellow solid; m.p.: $166-167{ }^{\circ} \mathrm{C} .{ }^{1} \mathrm{H}$ NMR $\left(300.1 \mathrm{MHz}, \mathrm{CDCl}_{3}\right): \delta 8.99-8.89(\mathrm{~m}, 1 \mathrm{H}), 8.56-8.44(\mathrm{~m}, 2 \mathrm{H}), 8.08$ (s, 1H), $7.94(\mathrm{~d}, J=7.5 \mathrm{~Hz}, 2 \mathrm{H}), 7.60-7.32(\mathrm{~m}, 4 \mathrm{H}), 4.08(\mathrm{~s}, 3 \mathrm{H}) .{ }^{13} \mathrm{C}\left\{{ }^{1} \mathrm{H}\right\} \mathrm{NMR}(75.5 \mathrm{MHz}$, $\left.\mathrm{CDCl}_{3}\right): \delta 167.1,152.1,150.7,143.5,136.0,132.4,131.3,131.1,130.3,128.5,128.1,126.7$, 124.9, 123.6, 123.1, 122.3, 61.9. IR (neat, $\mathrm{cm}^{-1}$ ) v: 3064, 3031, 2949, 1645, 1585, 1469, 1367 , 1257, 1168, 1080, 978, 876, 769, 690, 578. HRMS (ESI ${ }^{+}$): calcd for $\mathrm{C}_{19} \mathrm{H}_{14} \mathrm{ClN}_{2} \mathrm{O}_{2} \mathrm{~S} \mathrm{~m} / \mathrm{z}$ $369.0465[\mathrm{M}+\mathrm{H}]^{+}$, found $369.0461(-1.1 \mathrm{ppm})$.

Supporting Information: spectral data for all new compounds found in the SI.

\section{Acknowledgments}

This work was partially supported by INSA Rouen, Rouen University, CNRS, EFRD, Labex SynOrg (ANR-11-LABX-0029), Région Normandie (Crunch Network), the French National Research Agency (ANR-17-CE07-0038-01) and Innovation Chimie Carnot (I2C). T. B. thanks the European Research Council (ERC) under the European Union's Horizon 2020 research and innovation program (grant agreement no. 758710). M.-Y.C. thanks the French National Research Agency for a doctoral fellowship (ANR-17-CE07-0038-01).

\section{References}

1 For selected reviews, see: (a) Lyons, T. M.; Sanford, M. S. Palladium-catalyzed liganddirected C-H functionalization reactions. Chem. Rev. 2010, 110, 1147-1169. (b) Jazzar, R.; Hitce, J.; Renaudat, A.; Sofack-Kreutzer, J.; Baudoin, O. Functionalization of organic molecules by transition-metal-catalyzed $\mathrm{C}\left(\mathrm{sp}^{3}\right)-\mathrm{H}$ activation. Chem. Eur. J. 2010, 16, 26542672. (c) Engle, K.; Mei, T.-S.; Wasa, M,; Yu, J.-Q. Weak coordination as a powerful means for developing broadly useful $\mathrm{C}-\mathrm{H}$ functionalization reactions. Acc. Chem. Res. 2011, 45, 788802. (d) Kuhl, N.; Hopkinson, M. N.; Wencel-Delord, J.; Glorius, F. Beyond directing groups: transition-metal-catalyzed $\mathrm{C}-\mathrm{H}$ activation of simple arenes. Angew. Chem. Int. Ed. 2012, 51, 10236-10254. (e) Chen, Z.-K.; Wang, B.; Zhang, J.-T.; Yu, W.-L.; Liu, Z.-X.; Zhang, Y.-H. Transition metal-catalyzed $\mathrm{C}-\mathrm{H}$ bond functionalizations by the use of diverse directing groups. Org. Chem. Front. 2015, 2, 1107-1295. (f) Pototschnig G.; Maulide N.; Schnurch M. Direct Functionalization of C-H Bonds by Iron, Nickel, and Cobalt Catalysis. Chem. Eur. J. 2017, 23, 9206-9232. (g) Sambiagio, C.; Schonbauer, D.; Blieck, R.; Dao-Huy, T.; Pototschnig, G.; Schaaf, P.; Wiesinger, T.; Zai, M. F.; Wencel-Delord, J.; Besset, T.; Maes, B. U. W.; Schnurch, M. A comprehensive overview of directing groups applied in metal-catalysed C-H functionalisation chemistry. Chem. Soc. Rev. 2018, 47, 6603-6743. (h) Ma, C.; Fang, P.; Mei, T.-S. Recent advances in $\mathrm{C}-\mathrm{H}$ functionalization using electrochemical transition metal 
catalysis. ACS. Catal. 2018, 8, 7179-7189. (i) Xu, Y.; Dong, G.-B. sp ${ }^{3}$ C-H activation via exotype directing groups. Chem. Sci. 2018, 9, 1424-1432. (j) Gandeepan, P.; Muller, T.; Zell, D.; Cera, G.; Warratz, S.; Ackermann, L. 3d Transition Metals for C-H Activation. Chem. Rev. 2019, 119, 2192-2452. (k) For an issue on C-H bond activation, see: Chem. Rev. 2017, 117, $8481-9520$.

2 For selected reviews, see: (a) McMurray, L.; O’Hara, F.; Gaunt, M. J. Recent developments in natural product synthesis using metal-catalysed C-H bond functionalisation. Chem. Soc. Rev. 2011, 40, 1885-1898. (b) Yamaguchi, J.; Yamaguchi, A. D.; Itami, K. C-H Bond functionalization: emerging synthetic tools for natural products and pharmaceuticals. Angew. Chem. Int. Ed. 2012, 51, 8960-9009. (c) Chen, D. Y.-K.; Youn, S. W. C-H Activation: a complementary tool in the total synthesis of complex natural products. Chem. Eur. J. 2012, 18, 9452-9474. (d) Schonherr, H.; Cernak, T. Profound methyl effects in drug discovery and a call for new C-H methylation reactions. Angew. Chem. Int. Ed. 2013, 52, 12256-12267. (e) Cernak, T.; Dykstra, K. D.; Tyagarajan, S.; Vachal, P.; Krska, S. W. The medicinal chemist's toolbox for late stage functionalization of drug-like molecules. Chem. Soc. Rev. 2016, 45, 546. (f) Abrams, D. J.; Provencher, P. A.; Sorensen, E. J. Recent applications of C-H functionalization in complex natural product synthesis. Chem. Soc. Rev. 2018, 47, 8925-8967. For selected examples, see: (g) Brady, P. B.; Bhat, V. Recent applications of Rh- and Pd-catalyzed C( $\left.\mathrm{sp}^{3}\right)_{-}$ $\mathrm{H}$ functionalization in natural product total synthesis. Eur. J. Org. Chem. 2017, 5179-5190. (h) Dailler, D.; Danoun, G.; Baudoin, O. A general and scalable synthesis of aeruginosin marine natural products based on two strategic $\mathrm{C}\left(\mathrm{sp}^{3}\right)$-H activation reactions. Angew. Chem. Int. Ed. 2015, 54, $4919-4922$.

3 Wang, K.; Hu, F.; Zhang, Y.; Wang, J. Directing group-assisted transition-metal-catalyzed vinylic C-H bond functionalization. Sci. China. Chem. 2015, 58, 1252-1265 and references therein.

4 Gorsuch, S.; Bavetsias, V.; Rowlands, M. G.; Aherne, G. W.; Workman, P.; Jarman, M.; McDonald, E. Synthesis of isothiazol-3-one derivatives as inhibitors of histone acetyltransferases (HATs). Bioorg. Med. Chem. 2009, 17, 467-474.

5 Stimson, L.; Rowlands, M. G.; Newbatt, Y. M.; Smith, N. F.; Raynaud, F. I.; Rogers, P.; Bavetsias, V.; Gorsuch, S.; Jarman, M.; Bannister, A.; Kouzarides, T.; McDonald, E.; Workman, P.; Aherne, G. W. Isothiazolones as inhibitors of PCAF and p300 histone acetyltransferase activity. Mol. Cancer Ther. 2005, 4, 1521-1532.

6 (a) Lewis, S. N.; Miller, G. A.; Hausman, M.; Szamborski, E. C. Isothiazoles I: 4-Isothiazolin3-ones. A general synthesis from 3,3'-dithiodipropionamides. J. Heterocyclic. Chem. 1971, 8, 571-580. (b) Weiler, E. D.; Petigara, R. B.; Wolfersberger, M. H.; Miller, G. A. Isothiazoles IX. Halogenation of 2-substituted-4-isothiazolin-3-ones. J. Heterocycl. Chem. 1977, 14, 627630. (c) Bell, A. S.; Fishwick, C. W. G.; Reed, E. J. Facile palladium catalyzed functionalization of 1,2-isothiazoline-3-ones. Tetrahedron Lett. 1994, 35, 6551-6554. (d) Casoni, A.; Celentano, G.; Clerici, F.; Contini, A.; Gelmi, M. L.; Mazzeo, G.; Pellegrino, S.; Rosini, C. Enantioselective synthesis, chiroptical properties and absolute configuration of 3aminosubstituted isothiazole S-oxides. Tetrahedron: Asym. 2009, 20, 2247-2256. (e) Csakai, A.; Smith, C.; Davis, E.; Martinko, A.; Coulup, S.; Yin, H. Saccharin derivatives as inhibitors of interferon-mediated inflammation. J. Med. Chem. 2014, 57, 5348-5355. 
7 (a) Goerdeler, J.; Mittler, W. Synthese von 3-Hydroxy-, 3-Alkoxy- und 3-Aminoisothiazolen. Chem. Ber. 1963, 96, 944-954. (b) Derbala, H. A.; Hamad, A.-S. S.; El Said, W. A.; Hashem, A. I. Conversion of 3-aryl-5-phenyl-2(3H)-furanones into 3(2H)-isothiazolone derivatives. Phosphorus, Sulfur Silicon Relat. Elem. 2001, 175, 153-162. (c) Kandeel, K. A.; Youssef, A. S. A.; Abou-Elmagd, W. S. I.; Hashem, A. I. Behavior of 3-benzylamino-5-aryl2(3H)-furanones towards some nitrogen nucleophiles. J. Heterocyclic Chem. 2006, 43, 957962. (d) Youssef, A. S. A.; Kandeel, K. A.; Abou-Elmagd, W. S. I.; Hashem, A. I. Conversion of 5-aryl-3-phenylthio-2 $(3 H)$-furanones into some nitrogen- and sulphur-containing heterocycles. Phosphorus, Sulfur Silicon Relat. Elem. 2007, 182, 85-97.

8 Wright, S. W.; Petraitis, J. J.; Freimark, B.; Giannaras, J. V.; Pratta, M. A.; Sherk, S. R.; Williams, J. M.; Magolda, R. L.; Arner, E. C. 2,5-Diarylisothiazolone: novel inhibitors of cytokine-induced cartilage destruction. Bioorg. Med. Chem. 1996, 4, 851-858.

9 Dwivedi, V.; Rajesh, M.; Kumar, R.; Kant, R.; Reddy, M.-S. A stereoselective thiocyanate conjugate addition to electron deficient alkynes and concomitant cyclization to N,Sheterocycles. Chem. Commun. 2017, 53, 11060-11063.

10 (a) Xiong, H.-Y.; Besset, T.; Cahard, D.; Pannecoucke, X. Palladium(II)-catalyzed directed trifluoromethylthiolation of unactivated $\mathrm{C}\left(\mathrm{sp}^{3}\right)-\mathrm{H}$ bonds. J. Org. Chem. 2015, 80, 4204-4212. (b) Zhao, Q.; Tognetti, V.; Joubert, L.; Besset, T.; Pannecoucke, X.; Bouillon, J.-P.; Poisson, T. Palladium-catalyzed synthesis of 3-trifluoromethyl-substituted 1,3-butadienes by means of directed C-H bond functionalization. Org. Lett. 2017, 19, 2106-2109. (c) Zhao, Q.; Poisson, T.; Pannecoucke, X.; Bouillon, J.-P.; Besset, T. Pd-catalyzed diastereoselective trifluoromethylthiolation of functionalized acrylamides. Org. Lett. 2017, 19, 5106-5109.

11 See the supporting information for details.

12 Note that no product resulting either from the formation of a $\mathrm{C}-\mathrm{N}$ bond with the phthtalimide or from a direct cyanation was observed.

13 Qiu, J.-S.; Karmaker, P. G.; Yin, H.-Q.; Chen, F.-X. Enantioselective organocatalyzed direct $\alpha$-thiocyanation of cyclic $\beta$-ketoesters by $N$-thiocyanatophthalimide. Org. Lett. 2018, 20, 16001603.

14 Zhao, Q.; Chen, M.-Y.; Poisson, T.; Pannecoucke, X.; Bouillon, J.-P.; Besset, T. Pdcatalyzed trifluoromethylthiolation of unsaturated compounds: a general approach. Eur. J. Org. Chem. 2018, 6167-6175.

15 Thrimurtulu, N.; Dey, A.; Maiti, D.; Volla, C. M. R. Cobalt-catalyzed sp²-C-H activation: intermolecular heterocyclization with allenes at room temperature. Angew. Chem. Int. Ed. 2016, $55,12361-12365$.

16 Pan, J.-L.; Chen, C.; Ma, Z.-J.; Zhou, J.; Wang, L.-R.; Zhang, S.-Y. Stereoselective synthesis of $Z$-vinylsilanes via palladium-catalyzed direct intermolecular silylation of $\mathrm{C}\left(\mathrm{sp}^{2}\right)-\mathrm{H}$ bonds. Org. Lett. 2017, 19, 5216-5219.

17 Renata, H.; Wang, Z. J.; Kitto, R. Z.; Arnold, F. H. P450-catalyzed asymmetric cyclopropanation of electron-deficient olefins under aerobic conditions. Catal. Sci. Technol. 2014, 4, 3640-3643. 
18 Motati, R. D.; Uredi, D.; Watkins, E. B. A general method for the metal-free, regioselective, remote C-H halogenation of 8-substituted quinolines. Chem. Sci. 2018, 9, 1782-1788.

TOC Graphic<smiles>[R]C=C([R1])C(=O)Nc1ccc([R])c2cccnc12</smiles><smiles>N#CN1C(=O)c2ccccc2C1=O</smiles><smiles>[R]c1ccc(N2CC([R])C(=O)CS2)c2ncccc12</smiles>

Electrophilic SCN source 